\title{
Dynamical System Analysis and Optimal Control Measures of Lassa Fever Disease Model
}

\author{
Ifeanyi Sunday Onah $\mathbb{D}^{1},{ }^{1}$ Obiora Cornelius Collins $\mathbb{D}^{2}{ }^{2}$ \\ Praise-God Uchechukwu Madueme, ${ }^{1}$ and Godwin Christopher Ezike Mbah' \\ ${ }^{1}$ Department of Mathematics, University of Nigeria, Nsukka, Nigeria \\ ${ }^{2}$ Institute of Systems Science, Durban University of Technology, Durban 4000, South Africa \\ Correspondence should be addressed to Obiora Cornelius Collins; obiora.c.collins@gmail.com
}

Received 10 November 2019; Revised 3 March 2020; Accepted 12 March 2020; Published 29 April 2020

Academic Editor: Remi Léandre

Copyright (C) 2020 Ifeanyi Sunday Onah et al. This is an open access article distributed under the Creative Commons Attribution License, which permits unrestricted use, distribution, and reproduction in any medium, provided the original work is properly cited.

\begin{abstract}
Lassa fever is an animal-borne acute viral illness caused by the Lassa virus. This disease is endemic in parts of West Africa including Benin, Ghana, Guinea, Liberia, Mali, Sierra Leone, and Nigeria. We formulate a mathematical model for Lassa fever disease transmission under the assumption of a homogeneously mixed population. We highlighted the basic factors influencing the transmission of Lassa fever and also determined and analyzed the important mathematical features of the model. We extended the model by introducing various control intervention measures, like external protection, isolation, treatment, and rodent control. The extended model was analyzed and compared with the basic model by appropriate qualitative analysis and numerical simulation approach. We invoked the optimal control theory so as to determine how to reduce the spread of the disease with minimum cost.
\end{abstract}

\section{Introduction}

Lassa fever is a zoonotic acute viral illness caused by the Lassa virus. The host of Lassa virus is a rodent known as the multimammate rat (Mastomys natalensis). Transmission of Lassa virus to humans occurs most commonly through contact with food or household items that are contaminated with infected rodent urine or faeces. Infection can occur when Mastomys rodents shed the virus in urine and droppings, and direct contact with these materials, through touching contaminated objects, eating contaminated food, or exposure to open cuts or sores, can lead to infection $[1,2]$. This disease is endemic in parts of West Africa including Benin, Ghana, Guinea, Liberia, Mali, Sierra Leone, and Nigeria $[1,2]$.

The current Lassa fever outbreak in Nigeria is a serious health problem that is currently affecting humanity negatively. According to the World Health Organization, "from 1 January through 9 February 2020, 472 laboratory confirmed cases including 70 deaths (case fatality ratio $=14.8 \%$ ) have been reported in 26 out of 36 Nigerian states and the Federal Capital Territory" [3]. The WHO reported that "Lassa fever is endemic in Nigeria and the annual peak of human cases is usually observed during the dry season (December-April) following the reproduction cycle of the Mastomys rats in the wet season (May-June)" [3]. Statistics reveal that approximately $90-95 \%$ of human infections with Lassa fever in Nigeria are due to indirect exposure to (through food or household items contaminated by infected rats' urine and faeces) or direct contact with infected Mastomys rats [3]. According to Centers for Disease Control and Prevention (CDC) and World Health Organization (WHO) report, Lassa fever's yearly prevalence in West Africa is estimated at 100,000 to 300,000 , with approximately 5000 deaths $[1,2]$.

Mathematical model is a powerful tool that has been successfully used to investigate the dynamics of infectious diseases [4-11]. Some of the recent studies on Lassa fever disease dynamics that used mathematical model and other methods are presented below. Mariën et al. [12] considered experimental field data together with a mathematical model to evaluate rodent control for fight against Lassa fever disease. Musa et al. [13] studied the Lassa fever epidemics in Nigeria from 2016 to 2019 using a mathematical model. Zhao et al. [14] used different mathematical models (i.e., the Richards, three-parameter logistic, and Gompertz and 
Weibull growth models) to study the epidemiological features of Lassa fever epidemics in different Nigerian regions and quantify the association between the basic reproduction number and state rainfall. Specifically, they used Lassa fever surveillance data to fit the models and estimate the basic reproduction number and epidemic turning points in different regions at different time periods. Iacono et al. [15] used an innovative modeling approach to estimate the likely contribution of human-to-human transmission for Lassa fever disease. Akhmetzhanov et al. [16] examined the seasonal drivers of Lassa fever epidemics in Nigeria by considering a mathematical model to analyze the datasets of human infection, rodent population dynamics, and climatological variations and capture the underlying transmission dynamics. A comprehensive report of analysis of the epidemiologic and clinical aspects of the Lassa fever outbreak that occurred in Nigeria during January 1-May 6, 2018, was carried out by Ilori et al. [17]. A detailed reviewed on data pertaining to the massive wave of Lassa fever cases that occurred in Nigeria in 2018 was conducted by Ilori et al. [18]. Ajayi et al. [19] conducted a research on the Lassa fever epidemic in Nigeria by analyzing clinical, epidemiological, and laboratory data from surveillance records and hospital statistics during the outbreak. Another expository research on the Lassa fever outbreak in Nigeria was conducted by Roberts [20].

Presently, there are limited treatment options and no vaccine has been approved to prevent Lassa fever infection. As a result, Warner et al. [21] conducted a research where they discussed several vaccine candidates that have shown efficacy in animal models that could be advanced toward clinical trials. Current research on the search for Lassa fever vaccine can be found in [22-24]. Given that there are limited treatment options and no vaccine has been approved to prevent Lassa fever infection, the prevalence of Lassa fever in most poor countries or developing countries could be reduced through measures like proper sanitation or cleanliness, provision of adequate health facilities, better hygiene practice, and reducing breeding sites for infected rodents $[1,2]$. Consequently, effective means of reducing Lassa fever disease is by the use of control intervention measures such as external protection, quarantine, isolation, treatment of infected individuals, and reduction of Mastomys rats [25-30]. Therefore, in this study, we shall consider a mathematical model to investigate the dynamics and control intervention strategies for Lassa fever disease. Sometimes, even when these control strategies are available, the ability to fund it becomes a vital issue, especially in poor communities where there are limited resources. This can be attributed to the fact that the spread of Lassa fever is related to poverty, limited resources, uncleanliness, and low economic status [31]. Optimal control theory can give insight into the best control measure necessary to control the spread of Lassa fever with minimum cost $[32,33]$. In this study, we consider optimal control theory to investigate how to reduce the spread of Lassa fever with minimum cost.

In trying to understand and discuss the dynamics of Lassa fever, there are some essential factors that must be taken into consideration, and amongst them are sanitation, transmission medium, rodent control, effective treatment, quarantine, isolation, climatology factors or rainfall, and effect of economic background [25-30]. Understanding how the factors that influence Lassa fever disease are related so as to determine the dynamics of Lassa fever is challenging. In view of this, a number of approaches as shown above have been used to model the dynamics of Lassa fever disease dynamics. However, to the best of our understanding, none of the studies in the literature have considered multiple transmission pathways incorporating multiple control measures in a mathematical model to investigate the dynamics and optimal control measures for Lassa fever infection. Our work tends to fill this long existing vacuum.

The remaining part of this study is arranged as follows. We present a control-free model and analyze it in Section 2. Each of the single control measure model is presented and analyzed in Section 3. Detailed analysis of the external protection, isolation, treatment, and rodent control models is presented in Sections 3.1, 3.2, 3.3, and 3.4. The multiple control model (i.e., the model that portrayed all the control measures discussed) is presented, and its analysis is given in Section 4. Analysis of the optimal control theory is presented in Section 5. The paper is concluded with the numerical simulation in Section 6 and the discussion of result is given in Section 7.

\section{Lassa Fever Disease Model and Analysis}

This section of the work presents a Lassa fever disease model that demonstrates the Lassa fever disease dynamics for a homogeneous population without any control intervention measures. Analysis of this model will help to ascertain the effect of the control measures in the subsequent models.

2.1. Formulation of the Control-Free Model. In the formulation of this model, we considered the standard SIR model and we assumed a constant human and rodent population sizes $N(t)$ and $Z(t)$, respectively. We partitioned the total human population $N(t)$ into classes like susceptible humans $S(t)$, infected humans $I(t)$, and recovered humans $R(t)$ which gives that the total population of humans becomes $N(t)=S(t)+I(t)+R(t)$ and the total rodent population $Z(t)$ is partitioned as susceptible rodents $P(t)$ and infectious rodents $Q(t)$ such that $Z(t)=P(t)+Q(t)$. Humans are recruited into the susceptible class $S(t)$ by birth at a rate $\mu$. Susceptible humans $S(t)$ get infected with Lassa fever or enter the infected class through either contact with infected humans $I(t)$ or through contact with infectious rodents $Q(t)$ at rate $\beta$ and $\alpha$, respectively. Infected humans $I(t)$ recover at a rate $\rho$. We considered both direct human-tohuman contact and human-to-rodent contact because research has shown that Lassa fever transmission in most cases is as a result of contact between human and Mastomys natalensis (rodent) and also between humans [34]. Rodents on the other hand are recruited into the susceptible class by birth at a rate $\xi$. Susceptible rodents proceed to the infected class or become infected with the Lassa virus due to contact with another infected rodent $Q(t)$ at a rate $\phi$. Natural death 
in all human and rodent classes occurs at rate $\mu$ and $\xi$, respectively. Putting all these assumptions and descriptions together we obtain

$$
\begin{aligned}
\dot{S}(t) & =\mu N(t)-\beta S(t) I(t)-\alpha S(t) Q(t)-\mu S(t), \\
\dot{I}(t) & =\beta S(t) I(t)+\alpha S(t) Q(t)-(\mu+\rho) I(t), \\
\dot{R}(t) & =\rho I(t)-\mu R(t) \\
\dot{P}(t) & =\xi Z(t)-\phi P(t) Q(t)-\xi P(t), \\
\dot{Q}(t) & =\phi P(t) Q(t)-\xi Q(t) .
\end{aligned}
$$

Since $R(t)$ does not affect the dynamics of model (1), we will ignore the equation $(\dot{R}(t)=\rho I(t)-\mu R(t))$ in our analysis unless otherwise stated. Our model (1) is in the form of the model considered by Abdullahi et al. [35] who carried out sensitivity analysis of a Lassa fever deterministic mathematical model. This study will complements the work of Abdullahi et al. [35] who did not consider single control measures, multiple control measures, or optimal control theory in their work. We assume that all our chosen parameters are positive and the initial conditions of the variables are assumed as follows:

$$
\begin{aligned}
& S(0)>0, \\
& I(0) \geq 0, \\
& R(0) \geq 0, \\
& P(0)>0, \\
& Q(0) \geq 0 .
\end{aligned}
$$
region

All the solutions of model (1) will enter the feasible $\Phi=\{S, I, R, P, Q\} \in \mathbf{R}_{+}^{5}: S+I+R=N, P+Q=Z, \quad S, I \leq N$,

$$
R \leq \rho N / \mu, \quad P, Q \leq Z \text {. }
$$

By applying the local invariant set theorem [36, 37], we have that the region $\Phi$ is positively invariant. Thus, model (1) is mathematically and epidemiologically well posed in the region $\Phi$.

2.2. Basic Reproduction Number. The control-free model (1) has a unique disease-free equilibrium (DFE) given by

$$
\left(S^{0}, I^{0}, P^{0}, Q^{0}\right)=(N, 0, Z, 0) \text {. }
$$

We determined the basic reproduction number $\mathscr{R}_{0}$ of the control-free model (1) by the next generation matrix approach [38] which is given by

$$
\mathscr{R}_{0}=\max \left\{\mathscr{R}_{h}, \mathscr{R}_{r}\right\} \text {, }
$$

where $\mathscr{R}_{h}=(\beta N /(\mu+\rho))$ and $\mathscr{R}_{r}=\phi Z / \xi$. Epidemiologically, $\mathscr{R}_{h}$ is the basic reproduction number associated to humans while $\mathscr{R}_{r}$ is the basic reproduction number associated to rodents.
2.3. Stability Analysis of the Disease-Free Equilibrium. In determining the short-term dynamics of Lassa fever, it is important to investigate the stability of the disease-free equilibrium (DFE) [38]. The short-term dynamics of Lassa fever disease can be described by the stability about its disease-free equilibrium (DFE) [31].

The Jacobian matrix $J^{0}$ of model (1) evaluated at the DFE (4) is

$$
J^{0}=\left(\begin{array}{cccc}
-\mu & \beta N & 0 & \alpha N \\
0 & -(\mu+\rho)+\beta N & 0 & \alpha N \\
0 & 0 & -\xi & \phi Z \\
0 & 0 & 0 & -\xi+\phi Z
\end{array}\right) .
$$

The Jacobian matrix $J^{0}$ has four eigenvalues given by

$$
\begin{aligned}
& \lambda_{1}=-\mu, \\
& \lambda_{2}=(\mu+\rho)\left(\mathscr{R}_{h}-1\right), \\
& \lambda_{3}=-\xi, \\
& \lambda_{4}=\xi\left(\mathscr{R}_{r}-1\right) .
\end{aligned}
$$

Clearly, $\lambda_{1}<0$ and $\lambda_{3}<0$. Also, $\lambda_{2}<0$ if $\mathscr{R}_{h}<1$ and $\lambda_{4}<0$ if $\mathscr{R}_{r}<1$. Therefore, the following theorem holds.

Theorem 1. The DFE of the control-free model (1) is locally asymptotically stable if $\mathscr{R}_{0}<1$ and unstable if $\mathscr{R}_{0}>1$.

Epidemiologically, Theorem 1 implies that Lassa fever disease can be eradicated from the entire population if $\mathscr{R}_{0}<1$ and if the initial size of the infected population is in the region of attraction of the DFE (4). Otherwise, the disease will persist in the population if $\mathscr{R}_{0}>1$. We also need to investigate the global stability at DFE to show that eliminating the disease from the population is independent of the initial size of the infected population. In doing this, we will invoke the global stability result by Castillo-Chavez et al. [39].

Theorem 2. The DFE of the control-free model (1) is globally asymptotically stable if $\mathscr{R}_{0}<1$.

Proof. To prove this, we need just to show that the conditions $(\mathrm{H} 1)$ and $(\mathrm{H} 2)$ of the global stability result by Castillo-Chavez et al. [39] hold when $\mathscr{R}_{0}<1$. In our model (1), we have $X_{1}=(S, P), X_{2}=(I, Q)$ and $X_{1}^{*}=(N, Z)$. The systems

$$
\begin{aligned}
& \frac{\mathrm{d} X_{1}}{\mathrm{~d} t}=F\left(X_{1}, 0\right)=\mu N-\mu S, \\
& \frac{\mathrm{d} X_{1}}{\mathrm{~d} t}=F\left(X_{1}, 0\right)=\xi Z-\xi P,
\end{aligned}
$$

which are linear, and by solving, we have

$$
\begin{aligned}
& S(t)=N-(N-S(0)) e^{-\mu t}, \\
& P(t)=Z-(Z-P(0)) e^{-\xi t} .
\end{aligned}
$$


Taking the limits of the above equations gives $S(t) \longrightarrow N$ as $t \longrightarrow \infty$ and $P(t) \longrightarrow Z$ as $t \longrightarrow \infty$ regardless of the value of $S(0)$ and $P(0)$. Thus, $X_{1}^{*}=(N, Z)$ are globally asymptotically stable. Next, we have that

$$
G\left(X_{1}, X_{2}\right)=\left(\begin{array}{c}
\beta S I+\alpha S Q-(\mu+\rho) I \\
\phi P Q-\xi Q
\end{array}\right) .
$$

We get the value of $A$ and $A X$ as follows:

$$
\begin{gathered}
A=\left(\begin{array}{cc}
-(\mu+\rho)+\beta N & \alpha N \\
0 & -\xi+\phi Z
\end{array}\right), \\
A X=\left(\begin{array}{c}
(-(\mu+\rho)+\beta N) I+\alpha N Q \\
-\xi Q+\phi Z Q
\end{array}\right) .
\end{gathered}
$$

The matrix above is clearly an $M$ - matrix with nonnegative off diagonal elements. Hence, we find $\widehat{G}$ which is given as $A X-G$, so we have

$$
\widehat{G}\left(X_{1}, X_{2}\right)=\left(\begin{array}{c}
(N-S) \beta I+(N-S) \alpha Q \\
\phi Q(Z-P)
\end{array}\right) .
$$

Since $0 \leq S \leq N$ and $0 \leq P \leq Z$, it becomes obvious that $\widehat{G}\left(X_{1}, X_{2}\right) \geq 0$. This completes the proof.

The epidemiological implication of this result is that Lassa fever will be eradicated from the community irrespective of the initial population of infected provided $\mathscr{R}_{0}<1$.

2.4. Outbreak Growth Rate. If $\mathscr{R}_{0}>1$, then the DFE (4) becomes unstable and there is a possibility of disease outbreak occurring in the population. The positive (dominant) eigenvalue of the Jacobian at the DFE is typically referred to as the initial outbreak growth rate $[40,41]$. The eigenvalues of the Jacobian of model (1) evaluated at the DFE (4) are

$$
\begin{aligned}
& \lambda_{1}=-\mu, \\
& \lambda_{2}=(\mu+\rho)\left(\mathscr{R}_{h}-1\right), \\
& \lambda_{3}=-\xi, \\
& \lambda_{4}=\xi\left(\mathscr{R}_{r}-1\right) .
\end{aligned}
$$

From the above, it is evident that the positive (dominant) eigenvalues are given by

$$
\lambda^{+}=\max \left\{\lambda_{2}, \lambda_{4}\right\}
$$

Graphically, the value of $\lambda^{+}>0$ stands for the steepness of the increasing fraction curve [4]. This implies that the higher the value of $\lambda^{+}$, the more severe the disease outbreak. The epidemiological implication of this is that in the absence of control measure to reduce the spread of the infection such that $\mathscr{R}_{0}>1$, then there is a high tendency that an outbreak will spread to the entire population and will grow at a rate $\lambda^{+}$.

2.5. Stability Analysis of the Endemic Equilibrium. The longterm dynamics of a dynamical system model can be described by the stability about the endemic equilibrium (EE) [36]. So, we analyze the stability about the endemic equilibrium point. When $\mathscr{R}_{0}>1$, a unique EE occurs in the model and is given by

$$
\left(S^{e}, I^{e}, P^{e}, Q^{e}\right)=\left(\frac{\mu N}{\beta I^{e}+\alpha Q^{e}+\mu}, \frac{-b+\sqrt{b^{2}-4 a c}}{2 a}, \frac{Z}{\mathscr{R}_{r}}, P^{e}\left(\mathscr{R}_{r}-1\right)\right) \text {, }
$$

where $\quad a=\beta(\mu+\rho), b=(\mu+\rho)\left(\mu+\alpha Q^{e}\right)-\mu \beta N \quad$ and $c=-\alpha \mu N Q^{e}$.

Computing the Jacobian about (1), we have

$$
J=\left[\begin{array}{cccc}
-\mu-\beta I^{e}-\alpha Q^{e} & -\beta S^{e} & 0 & -\alpha S^{e} \\
\beta I^{e}+\alpha Q^{e} & \beta S^{e} e-\mu-\rho & 0 & \alpha S e \\
0 & 0 & -\xi-\rho Q^{e} & -\xi P^{e} \\
0 & 0 & \rho Q^{e} & \rho P^{e}-\xi
\end{array}\right] .
$$

From (16), solving for eigenvalues, we have

$$
\begin{aligned}
& \lambda_{1,2}=\frac{-a_{44} \pm \sqrt{a_{44}^{2}-4 a_{34} a_{43}}}{2}, \\
& \lambda_{3,4}=\frac{a_{22}-a_{11} \pm \sqrt{\left(a_{11}+a_{22}\right)^{2}-4 a_{12} a_{21}}}{2} .
\end{aligned}
$$

We can easily see that $\lambda_{1,2}<0$, since $a_{44}=\xi+\rho Q>0$, $a_{34}=\xi P^{e}>0$, and $a_{43}=\rho Q e>0$. Similarly, $\lambda_{3,4}<0$, since $a_{12}=\beta S^{e}>0, \quad a_{21}=\beta I^{e}+\alpha Q^{e}>0, \quad$ and $\quad a_{11}=\mu+\beta I^{e}+$ $\alpha Q^{e}>0$. Choosing $\mathscr{R}_{h}=\left(N / S^{e}\right)>1$ gives $a_{22}=\beta S^{e}-(\mu+$ $\rho)=(\mu+\rho)\left[\left(\beta S^{e} N /((\mu+\rho) N)\right)-1\right]=(\mu+\rho)\left[\mathscr{R}_{h}\left(S^{e} / N\right)-\right.$ $1]=0$, which completes the verifications that all the eigenvalues are negative. Based on this, we obtained the theorem that follows.

Theorem 3. The unique EE (15) is locally asymptotically stable whenever $\mathscr{R}_{h}=\left(N / S^{e}\right)>1$ and $\mathscr{R}_{r}>1$.

Note that when $\mathscr{R}_{0}=1$, i.e., $\mathscr{R}_{h}=\mathscr{R}_{r}=1$, then the disease-free equilibrium and the endemic equilibrium coincide. This suggests that $\mathscr{R}_{0}=1$ is a bifurcation point. Therefore, the interest will be to make $\mathscr{R}_{0} \leq 1$ so that there will be more chances of eliminating Lassa fever from the population. Further investigation on this will be done in our subsequent analysis particularly in our numerical simulations.

\section{Single Control Models}

In this section, we investigate the impact of each single control measures. Specifically, we shall discuss the impact of external protection, isolation, treatment, and rodent control measures analytically in the subsequent sections using appropriate control model.

3.1. External Protection Model. Lassa fever transmission in humans can be from one human to another human through contaminated medical equipment or through direct contact as a result of skin break [25]. With these information above, we can deduce that it can be transmitted through any kind of body contact where fluids are transferred. In this work, we 
refer to any external protection against exchange of body fluids as external protection. This external protection can be informed of complete use of personal protective equipment (PPE) especially by health workers in case of an endemic environment. In our health centers or specialist hospitals where Lassa fever is being treated, there should be relevant training on how to don and doff the PPE by the health officers. Adequate supervision of health officers with regards to using the PPE is crucial. The use of PPE should not only be restricted to the doctors or nurses, but even the cleaners and ward aides should make use of PPE in their services because most times they come in contact with the patients' wastes or even in handling the deceased patients. The use of condom can also be seen as one of the control measures in reducing the spread of some infectious disease such as Lassa fever. To determine the effects of condom use and PPE which we refer to as external protection in reducing the spread of Lassa fever, we extend model (1) by assuming that susceptible humans use condom and PPE at the rate $\tau$ with the efficiency of the condom and PPE given as $e$, and the contact rate of infectious human and susceptible humans using condom and PPE is also $\phi_{h 1}$. Based on the above assumptions, we obtain the model

$$
\begin{aligned}
\dot{S}(t) & =\mu N(t)-\beta S(t) I(t)-\alpha S(t) Q(t)-(\mu+\tau) S(t), \\
\dot{G}(t) & =\tau S(t)-(1-e) \beta G(t) I(t)-\mu G(t), \\
\dot{I}(t) & =\beta S(t) I(t)+\alpha S(t) Q(t)+(1-e) \beta G(t) I(t)-(\mu+\rho) I(t), \\
\dot{R}(t) & =\rho I(t)-\mu R(t), \\
\dot{P}(t) & =\xi Z(t)-\phi P(t) Q(t)-\xi P(t), \\
\dot{Q}(t) & =\phi P(t) Q(t)-\xi Q(t),
\end{aligned}
$$

which is an extension of model (1), adding a class of humans using external protection. The variable $G(t)$ represents susceptible humans using external protection at time $t$.

3.1.1. Analysis of the External Protection Model. The DFE of model (18) when external protection is introduced is given by

$$
\left(S_{e}^{0}, G_{e}^{0}, I_{e}^{0}, P_{e}^{0}, Q_{e}^{0}\right)=\left(\frac{\mu N}{\mu+\tau}, \frac{\tau N}{\mu+\tau}, 0, Z, 0\right),
$$

and the corresponding basic reproduction number is

$$
\mathscr{R}_{0}^{e}=\max \left\{\frac{\beta\left(S_{e}^{0}+(1-e) G_{e}^{0}\right)}{\mu+\rho}, \mathscr{R}_{r}\right\} .
$$

where $\mathscr{R}_{h}^{e}=\left(\beta\left(S_{e}^{0}+(1-e) G_{e}^{0}\right) /(\mu+\rho)\right)$ and $\mathscr{R}_{r}^{e}=\phi Z / \xi$. This threshold $\mathscr{R}_{0}^{e}$ is the basic reproduction number when external protection is introduced. The value of threshold quantity $\mathscr{R}_{r}^{e}$ is not affected (i.e., $\mathscr{R}_{r}^{e}=\mathscr{R}_{r}$ ) when external protection is considered as a control measure. A possible explanation could be that external protection targets only reducing humans' exposure to the disease and does not directly affect the rodent population. Note that $S_{e}^{0}+G_{e}^{0}=N$. The following inequity holds

$$
\begin{aligned}
& e<1 \Longleftrightarrow \mathscr{R}_{0}^{e}<\mathscr{R}_{0}, \quad \forall 0<e, \\
& e=0 \Longleftrightarrow \mathscr{R}_{0}^{e}=\mathscr{R}_{0}, \quad \text { for } e=0 .
\end{aligned}
$$

One can verify these equations by simple elementary algebraic manipulation. Equation (21) implies that external protection decreases the number of secondary infected humans by a rate $e$ or in other words, use of external protection decreases $\mathscr{R}_{0}$ by a rate $e$. The parameter $e=0$ means that external protection has no effect or is useless [42] while $\tau=0$ means that no susceptible individual is using external protection. This information above implies that external protection use exerts some impact in an attempt of reducing secondary infections in the human population provided $0<e$ and $\tau \leq 1$.

Also, the quantity $e$ measures the effectiveness of use of external protection as a control intervention strategy in reducing the spread of Lassa fever. Since $\mathscr{R}_{0}-\mathscr{R}_{0}^{e}=\mathscr{R}_{0}(1-e)$ and $0<e \leq 1$, then $e \longrightarrow 1$ means that external protection has great effect while $e \longrightarrow 0$ means that external protection has no effect. Therefore, we can express the effectiveness of use of external protection $e$ in percentage as [4]

$$
E_{p}^{0}=(1-e) \times 100
$$

We can infer now that use of external protection plays a vital role in bringing down the possible number of secondary infections by $G^{0}$ percent. We proceed to investigate the dynamics of Lassa fever disease in its short term with the use of external protection. The stability of the external protection model investigated at DFE summarized in the theorem below.

Theorem 4. The DFE of model (18) when external protection is introduced is both locally and globally asymptotically stable provided that $\mathscr{R}_{0}^{e}<1$.

We can prove Theorem 4 using the approach that is similar to that of Theorem 2 and a stability result from Theorem 2 of Van den Driessche and Watmough [38]. The epidemiological implication of Theorem 4 is that Lassa fever can be eradicated in the entire human population if they make use of external protection, provided $\mathscr{R}_{0}^{e}<1$. We have previously shown that infections can be eradicated in the absence of control measures provided $\mathscr{R}_{0}<1$. And since $\mathscr{R}_{0}^{e}<\mathscr{R}_{0}<1$, we can deduce from above that introducing use of external protection will lead to faster eradication of the outbreak. However, if the external protection is not effective enough such that $\mathscr{R}_{0}^{e}>1$, then Lassa fever may remain in the population in the presence of external protection. To investigate the long-term dynamics of Lassa fever when external protection is considered, we conduct the stability analysis of the external protection model about the endemic equilibrium.

When $\mathscr{R}_{0}^{e}>1$, an endemic equilibrium occurs in the external protection model (18). For the endemic equilibrium, we consider a situation when $e=1$ (i.e., when the that external protection has great effect). For this case, the endemic equilibrium of the external protection model (18) becomes 


$$
\left(S^{e}, G^{e}, I^{e}, P^{e}, Q^{e}\right)=\left(\frac{\mu N}{\beta I^{e}+\alpha Q^{e}+\mu}, \frac{\tau S^{e}}{\mu}, \frac{-b+\sqrt{b^{2}-4 a c}}{2 a}, \frac{Z}{\mathscr{R}_{r}}, P^{e}\left(\mathscr{R}_{r}-1\right)\right)
$$

where $a=\beta(\mu+\rho), b=(\mu+\rho)\left(\mu+\tau+\alpha Q^{e}\right)-\mu \beta N$, and $c=-\alpha \mu N Q^{e}$. To show that the external protection model (18) is stable about the endemic equilibrium (24), we must show that all the eigenvalues of Jacobian of the model evaluated at EE have negative real parts. By similar mathematical analysis, we have that for $\left(\mathscr{R}_{h}^{e}=\left(N / S^{e}\right)>1, \mathscr{R}_{r}>1\right.$, and $e=1)$, the eigenvalues of Jacobian of the external protection model (18) evaluated at EE (18) are

$$
\begin{aligned}
\lambda_{1} & =-\mu, \\
\lambda_{2,3} & =\frac{a_{11} \pm \sqrt{a_{11}^{2}+4 a_{13} a_{31}}}{2}, \\
\lambda_{4,5} & =\frac{a_{44} \pm \sqrt{a_{44}^{2}+4 a_{45} a_{54}}}{2},
\end{aligned}
$$

where $a_{11}=-\left(\mu+\tau+\beta I^{e}+\alpha Q^{e}\right), a_{13}=-\beta S^{e}, a_{31}=\beta I^{e}+$ $\alpha Q^{e}, a_{44}=-\left(\xi+\phi Q^{e}\right), a_{45}=-\phi P^{e}$, and $a_{54}=\phi Q^{e}$. Clearly, we can see that all the eigenvalues have negative real parts. Based on this, we obtained the following theorem.

Theorem 5. The endemic equilibrium (24) of the external protection model (18) is locally asymptotically stable whenever $\mathscr{R}_{h}^{e}=\left(N / S^{e}\right)>1, \mathscr{R}_{r}>1$, and $e=1$.

This theorem implies that if the introduction of external protection is not effective enough such that $\mathscr{R}_{0}^{e}>1$, then the disease will persist in the population.

3.2. Isolation Model. Vaccination, quarantine, and isolation have been a major tool now in most mathematical models in trying to control infectious diseases effectively. This has been applied to most known infectious diseases and can be said to be a tool for diseases yet to be fully identified [27-30]. Having identified this fact that vaccine and isolation is a good control measure for infectious diseases, we first investigate the availability of vaccine. If there exists vaccine for such infectious disease, then it is important to create a vaccinated or an isolated class to enable one do a good study on its impact [30]. In our study, we describe isolation as the removal of patients that have been detected to show clear sign of the infection so as not to infect other persons and for possible and effective treatment. When humans are isolated, one can regulate their treatment and ensure that there is minimal contact with other humans in the population. With this information, it comes to mind that isolation will be a good control measure in curbing the spread of Lassa fever. In doing this, we introduce isolation in our control-free model (1) with the assumption that infected humans are isolated at a rate $\sigma$ (where $0 \leq \sigma \leq 1$ ) and isolated humans recover at rate $\rho_{f}$ and are treated at rate $v$ to obtain the isolated model as

$$
\begin{aligned}
& \dot{S}(t)=\mu N(t)-\beta S(t) I(t)-\alpha S(t) Q(t)-\mu S(t), \\
& \dot{I}(t)=\beta S(t) I(t)+\alpha S(t) Q(t)-(\mu+\rho+\sigma) I(t), \\
& \dot{F}(t)=\sigma I(t)-\left(\mu+\delta+\rho_{f}+v\right) F(t), \\
& \dot{R}(t)=\rho_{f} F(t)+\rho I(t)-\mu R(t) \\
& \dot{P}(t)=\xi Z(t)-\phi P(t) Q(t)-\xi P(t), \\
& \dot{Q}(t)=\phi P(t) Q(t)-\xi Q(t)
\end{aligned}
$$

where $F$ is the isolated population.

3.2.1. Analysis of the Isolation Model. The DFE of model (26) when isolation is introduced is given by

$$
\left(S^{0}, I^{0}, F^{0}, P^{0}, Q^{0}\right)=(N, 0,0, Z, 0)
$$

and the corresponding basic reproduction number is

$$
\mathscr{R}_{0}^{f}=\max \left\{\frac{\beta N}{\mu+\rho+\sigma}, \frac{\phi Z}{\xi}\right\},
$$

where $\mathscr{R}_{h}^{f}=\beta N /(\mu+\rho+\sigma)$ and $\mathscr{R}_{r}^{f}=\phi Z / \xi$. The threshold quantity $\mathscr{R}_{0}^{f}$ stands for the basic reproduction number in the presence of isolation. Note that $\mathscr{R}_{r}^{f}=\mathscr{R}_{r}$. A possible reason for this is because isolation targets only the human population, the basic reproduction number associated with the rodents is not directly affected. Clearly, we deduce that the following inequalities hold:

$$
\begin{gathered}
\sigma \leq 1 \Longleftrightarrow \mathscr{R}_{0}^{f}<\mathscr{R}_{0}, \\
\sigma=0 \Longleftrightarrow \mathscr{R}_{0}^{f}=\mathscr{R}_{0} .
\end{gathered}
$$

This epidemiologically suggests that isolation plays a role in curbing the increase of secondary infections in the population provided $0<\sigma \leq 1$. We investigate the stability of the isolation model at DFE to see the possible dynamics of the model in short term. The results of our analysis is presented in the theorem below.

Theorem 6. The DFE of the isolated model (26) is both locally and globally asymptotically stable provided $\mathscr{R}_{0}^{f}<1$.

The biological implication of Theorem 6 is that Lassa fever can be eradicated from the population through isolating the infected humans whenever $\mathscr{R}_{0}^{f}<1$. However, if infected humans are not properly isolated such that $\mathscr{R}_{0}^{f}>1$, then the disease may remain endemic in the population.

When $\mathscr{R}_{0}^{f}>1$, a unique EE occurs in the isolation model (26) and is given by 


$$
\begin{aligned}
& \left(S^{e}, I^{e}, F^{e}, P^{e}, Q^{e}\right)=\left(\frac{\mu N}{\beta I^{e}+\alpha Q^{e}+\mu}, \frac{-b+\sqrt{b^{2}-4 a c}}{2 a}, \frac{\sigma I^{e}}{\mu+\delta+\rho_{f}+v}, \frac{Z}{\mathscr{R}_{r}}, P^{e}\left(\mathscr{R}_{r}-1\right)\right), \\
& \quad \mathscr{R}_{0}^{t}=\left\{\frac{\beta N}{\mu+\rho+\eta}, \frac{\phi Z}{\mu}\right\},
\end{aligned}
$$

where $a=\beta(\mu+\rho+\sigma), b=(\mu+\rho+\sigma)\left(\mu+\alpha Q^{e}\right)-\mu \beta N$, and $c=-\alpha \mu Q^{e} N$. The results of the stability analysis about the endemic equilibrium of the isolation model (26) are summarized in the theorem below.

Theorem 7. The unique EE (31) is locally asymptotically stable whenever $\mathscr{R}_{h}^{f}=\left(N / S^{e}\right)>1$ and $\mathscr{R}_{r}>1$.

Theorem 7 can be established using a similar approach in the proof of Theorem 3. This result implies that if $\mathscr{R}_{0}^{f}>1$, the disease will persist in the community in the presence of isolation as a control measure.

3.3. Treatment Model. Most infectious diseases are highly reduced if there is an effective treatment. Lassa fever shares similar symptoms with many other known viral hemorrhagic fevers, including that of Ebola virus, dengue fever, malaria, and typhoid fever [43]. Hospitalized patients have recorded high fatality rates of Lassa fever; this rate ranges between $20 \%-40 \%$, but from serologic data it was reported that mild or sub clinical infections are likely to occur [44]. From the literature, it is seen that treatment of humans or patients infected with Lassa fever has been to a large extent symptomatic and supportive; specific treatment has been attempted in a small number of patients to whom Lassa-immune plasma was administered, with equivocal success [26]. The antiviral drug that has been proven to be effective when administered at the early stage of the disease is ribavirin [43]. Interestingly, infected humans when they recover from illness acquire permanent immunity. With this information, it comes to mind that effective treatment will be a good control strategy; hence, it is important to investigate how to decrease the spread of Lassa fever by applying treatment as a control intervention strategy. By doing this, we introduce treatment in the control-free model (1) by assuming that infected humans are treated at rate $\eta$ (where $0 \leq \eta \leq 1$ ) and treated humans $T(t)$ recover at rate $\rho_{t}$ to obtain the treatment model as

$$
\begin{aligned}
\dot{S}(t) & =\mu N(t)-\beta S(t) I(t)-\alpha S(t) Q(t)-\mu S(t), \\
\dot{I}(t) & =\beta S(t) I(t)+\alpha S(t) Q(t)-(\mu+\rho+\eta) I(t), \\
\dot{T}(t) & =\eta I(t)-\left(\mu+\rho_{t}\right) T(t), \\
\dot{R}(t) & =\rho I(t)+\rho_{t} T(t)-\mu R(t), \\
\dot{P}(t) & =\xi Z(t)-\phi P(t) Q(t)-\xi P(t), \\
\dot{Q}(t) & =\phi P(t) Q(t)-\xi Q(t) .
\end{aligned}
$$

3.3.1. Analysis of the Treatment Model. The DFE of the treatment model (32) is given by

$$
\left(S^{0}, I^{0}, T^{0}, P^{0}, Q^{0}\right)=(N, 0,0, Z, 0),
$$

and the treatment reproduction number is where $\mathscr{R}_{h}^{t}=\beta N /(\mu+\rho+\eta)$ and $\mathscr{R}_{r}^{t}=\phi Z / \mu$. Note that $\mathscr{R}_{r}^{t}=\mathscr{R}_{r}$. A possible reason for this is because treatment targets only the infected humans, the basic reproduction number associated with the rodents is not affected directly. The threshold quantity $\mathscr{R}_{0}^{t}$ stands for the basic reproduction number in the presence of treatment. So, we can clearly deduce that the following inequality holds:

$$
\begin{gathered}
\eta \leq 1 \Longleftrightarrow \mathscr{R}_{0}^{t}<\mathscr{R}_{0}, \\
\eta=0 \Longleftrightarrow \mathscr{R}_{0}^{t}=\mathscr{R}_{0} .
\end{gathered}
$$

This suggests that treatment of infected individuals plays a role in decreasing the increase of secondary infections in the population provided $0<\tau \leq 1$. Next, we investigate the stability of the treatment model about DFE to see the possible dynamics of the model in short term which is presented in the theorem below.

Theorem 8. The DFE of the treatment model (32) is both locally and globally asymptotically stable, provided $\mathscr{R}_{0}^{t}<1$.

Biologically speaking, Theorem 8 implies that Lassa fever can be eradicated from the population through treatment of infected individuals whenever $\mathscr{R}_{0}^{t}<1$. However, if individuals are not properly treated such that $\mathscr{R}_{0}^{t}>1$, then the disease persists in the population.

By a similar approach as in the isolation model, we have that if $\mathscr{R}_{0}^{t}>1$, a unique EE occurs in the treatment model (32) and is given by

$$
\begin{aligned}
\left(S^{e}, I^{e}, T^{e}, P^{e}, Q^{e}\right)= & \left(\frac{\mu N}{\beta I^{e}+\alpha Q^{e}+\mu}, \frac{-b+\sqrt{b^{2}-4 a c}}{2 a},\right. \\
& \left.\frac{\eta I^{e}}{\mu+\rho_{t}}, \frac{Z}{\mathscr{R}_{r}}, P^{e}\left(\mathscr{R}_{r}-1\right)\right),
\end{aligned}
$$

where $a=\beta(\mu+\rho+\eta), b=(\mu+\rho+\eta)\left(\mu+\alpha Q^{e}\right)-\mu \beta N$, and $c=-\alpha \mu \mathrm{Q}^{e} N$. The results of the stability analysis about the endemic equilibrium of the treatment model (32) are summarized in the theorem below.

Theorem 9. The unique EE (37) is locally asymptotically stable whenever $\mathscr{R}_{h}^{t}=\left(N / S^{e}\right)>1$ and $\mathscr{R}_{r}>1$.

Theorem 9 can be established using a similar approach in the proof of Theorem 3. This result implies that if $\mathscr{R}_{0}^{t}>1$, the disease will persist in the population in the presence of treatment of infected individuals as a control measure.

3.4. Rodent Control Model. According to a report from the Centers for Disease Control and Prevention (CDC) [45], Lassa 
fever is transmitted through contact between humans and infected rodents of the genus Mastomys known as Mastomys natalensis, as a result of poor hygiene, poor sanitation, and unhealthy human practices. Lassa fever cases can be reduced if there is a drastic reduction in the infected rat population, which also will lead to rare contact between human and infected rats through proper and regular sanitation and better hygiene practices. To determine the effects of rodent control as a control intervention strategy, we extend model (1) by assuming that the control rate of infected rodents as $c$ to obtain

$$
\begin{aligned}
& \dot{S}(t)=\mu N(t)-\beta S(t) I(t)-\alpha S(t) Q(t)-\mu S(t), \\
& \dot{I}(t)=\beta S(t) I(t)+\alpha S(t) Q(t)-(\mu+\rho) I(t), \\
& \dot{R}(t)=\rho I(t)-\mu R(t), \\
& \dot{P}(t)=\xi Z(t)-\phi(1-c) P(t) Q(t)-\xi P(t), \\
& \dot{Q}(t)=\phi(1-c) P(t) Q(t)-\xi Q(t),
\end{aligned}
$$

where $0 \leq c \leq 1$.

3.4.1. Analysis of the Rodent Control Model. The DFE of model (38) when rodent control is introduced is given by

$$
\left(S^{0}, I^{0}, P^{0}, Q^{0}\right)=(N, 0, Z, 0),
$$

and the corresponding basic reproduction number is

$$
\mathscr{R}_{0}^{c}=\left\{\frac{\beta N}{\mu+\rho}, \frac{\phi(1-c) Z}{\xi}\right\},
$$

which can be written as

$$
\mathscr{R}_{0}^{c}=\left\{\mathscr{R}_{h}, \mathscr{R}_{r}(1-c)\right\},
$$

where $\mathscr{R}_{r}^{c}=\mathscr{R}_{r}(1-c)$ and $\mathscr{R}_{h}=\mathscr{R}_{h}^{c}$. Note that the basic reproduction number associated with human is unaffected (i.e., $\mathscr{R}_{h}=\mathscr{R}_{h}^{c}$ ) when rodent control is considered. A possible explanation for this is that rodent control does not directly affect human population. The threshold quantity $\mathscr{R}_{0}^{c}$ stands for the basic reproduction number when rodent control is considered. Similarly, we obtain that

$$
\begin{gathered}
c<1 \Longleftrightarrow \mathscr{R}_{0}^{c}<\mathscr{R}_{0}, \\
c=0 \Longleftrightarrow \mathscr{R}_{0}^{c}=\mathscr{R}_{0} .
\end{gathered}
$$

This suggests that rodent control plays an important role in decreasing the number of secondary infections in the population given that $0<c \leq 1$. However, to investigate the shortterm dynamics of Lassa fever considering rodent control, it is of importance to investigate the stability of the rodent control model at DFE which is presented in the theorem below.

Theorem 10. The DFE of the rodent control model (38) is locally and globally asymptotically stable, whenever $\mathscr{R}_{0}^{c}<1$.

Epidemiologically, Theorem 10 means that Lassa fever disease can be eliminated from the population through rodent control whenever $\mathscr{R}_{0}^{c}<1$. On the contrary, if rodent control is not effective enough such that $\mathscr{R}_{0}^{c}>1$, then a unique EE occurs in the rodent control model (38) and is given by

$$
\left(S^{e}, I^{e}, P^{e}, Q^{e}\right)=\left(\frac{\mu N}{\beta I^{e}+\alpha Q^{e}+\mu}, \frac{-b+\sqrt{b^{2}-4 a c}}{2 a}, \frac{Z}{\mathscr{R}_{r}^{c}}, P^{e}\left(\mathscr{R}_{r}^{c}-1\right)\right),
$$

where $\quad a=\beta(\mu+\rho), b=(\mu+\rho)\left(\mu+\alpha Q^{e}\right)-\mu \beta N, \quad$ and $c=-\alpha \mu Q^{e} N$. The results of the stability analysis about the endemic equilibrium of the rodent control model (38) are summarized in the theorem below.

Theorem 11. The unique EE (44) is locally asymptotically stable whenever $\mathscr{R}_{h}^{c}=\left(N / S^{e}\right)>1$ and $\mathscr{R}_{r}^{c}>1$.

Similarly, Theorem 11 can be established using the approach in the proof of Theorem 3 . This result implies that if $\mathscr{R}_{0}^{c}>1$, the disease will persist in the population when rodent control is considered as a control measure.

\section{Multiple Control Intervention Strategy Model}

We have seen that considering single control intervention strategy of each of the above control measures plays an important role in decreasing the secondary cases of Lassa fever infection in the entire human and rodent population. In this section, we consider the multiple control intervention strategy, that is, combining all the single control intervention strategies earlier discussed. So, we introduce the control strategies into model (1) simultaneously and obtain

$$
\begin{aligned}
\dot{S}(t)= & \mu N(t)-\beta S(t) I(t)-\alpha S(t) Q(t)-(\mu+\tau) S(t), \\
\dot{G}(t)= & \tau S(t)-(1-e) \beta G(t) I(t)-\mu G(t), \\
\dot{I}(t)= & \beta S(t) I(t)+\alpha S(t) Q(t)+(1-e) \beta G(t) I(t) \\
& -(\mu+\rho+\eta+\sigma) I(t), \\
\dot{F}(t)= & \sigma I(t)-\left(\mu+\delta+\rho_{f}+v\right) F(t) \\
\dot{T}(t)= & \eta I(t)+v F(t)-\left(\mu+\rho_{t}\right) T(t) \\
\dot{R}(t)= & \rho I(t)+\rho_{t} T(t)+\rho_{f} F(t)-\mu R(t), \\
\dot{P}(t)= & \xi Z(t)-\phi(1-c) P(t) Q(t)-\xi P(t), \\
\dot{Q}(t)= & \phi(1-c) P(t) Q(t)-\xi Q(t) .
\end{aligned}
$$

All the solutions of model (45) enter the feasible region

$$
\begin{aligned}
\Phi_{m}= & \left\{(S, G, I, T, F, R, P, Q) \in \mathbb{R}_{+}^{8}: S \leq S^{0}, E \leq G^{0}, I \leq N, T\right. \\
& \left.\leq T^{0}, F \leq F^{0}, R \leq R^{0}, P \leq P^{0}, Q \leq Z\right\},
\end{aligned}
$$

where $N=S+G+I+T+F+R, Z=P+Q, T^{0}=\eta N /(\mu+$ $\left.\rho_{t}\right), \quad F^{0}=\sigma N /\left(\mu+\delta+\rho_{f}+v\right), \quad$ and $\quad R^{0}=(\rho N / \mu)+$ $\eta N /\left(\mu\left(\mu+\rho_{t}\right)\right)$. The region $\Phi_{m}$ is positively invariant, and by this, we can proceed in determining the solution of model (45) in $\Phi_{m}$.

4.1. Analysis of the Multiple Control Intervention Strategy Model. The DFE of the multiple control intervention measure model (45) is given as 


$$
\left(S_{m}^{0}, G_{m}^{0}, I_{m}^{0}, F_{m}^{0}, T_{m}^{0}, P_{m}^{0}, Q_{m}^{0}\right)=\left(\frac{\mu N}{\mu+\tau}, \frac{\tau N}{\mu+\tau}, 0,0,0, Z, 0\right)
$$

and the basic reproduction number is given by

$$
\mathscr{R}_{0}^{m}=\max \left\{\frac{\beta S_{m}^{0}+(1-e) \beta G_{m}^{0}}{\mu+\rho+\eta+\sigma}, \frac{\phi(1-c) Z}{\xi}\right\},
$$

where $\quad \mathscr{R}_{h}^{m}=\left(\beta S_{m}^{0}+(1-e) \beta G_{m}^{0} /(\mu+\rho+\eta+\sigma)\right) \quad$ and $\mathscr{R}_{r}^{m}=(\phi(1-c) Z) / \xi$. The threshold quantity $\mathscr{R}_{0}^{m}$ stands for the basic reproduction number that takes into cognizance measures like external protection, quarantine, isolation, treatment, and rodent control. From equation (48), we have that

$$
e, c, \eta, \sigma<1 \Longleftrightarrow \mathscr{R}_{0}^{m}<\mathscr{R}_{0} .
$$

This suggests that multiple control intervention measures decrease the secondary infections. By doing so, we can actually express the effect of our control intervention strategies which are in equations (21), (29), (35), (42), and (49) in their compact form as

$$
\mathscr{R}_{0}^{m}<\mathscr{R}_{0}^{e}, \mathscr{R}_{0}^{f}, \mathscr{R}_{0}^{t}, \mathscr{R}_{0}^{c}<\mathscr{R}_{0} .
$$

This inequality shows that even though each of the single control has some impact in reducing the spread of Lassa fever, the multiple control has the greatest impact in reducing the spread of the disease. The short-term dynamics of the model when the multiple control measures are introduced are summarized in the theorem below.

Theorem 12. If $\mathscr{R}_{0}^{m}<1$, the DFE (47) of model (45) is globally asymptotically stable and unstable if $\mathscr{R}_{0}^{m}>1$.

The proof of Theorem 12 can be established using as similar approach as in the proof of Theorem 6. Epidemiologically, this implies that Lassa fever disease will be reduced to the barest minimum in the entire population, when we consider the multiple control intervention technique provided $\mathscr{R}_{0}^{m}<1$. Suppose that the multiple control intervention technique is not effective enough such that $\mathscr{R}_{0}^{m}>1$, then the disease might become endemic in the population. To investigate long-term dynamics of the disease, we conduct the stability analysis of the multiple control intervention model about the endemic equilibrium. By using similar assumptions as in the previous model we have that when $\mathscr{R}_{0}^{m}>1$, an endemic equilibrium occurs and is given by

$$
\left(S^{e}, G^{e}, I^{e}, F^{e}, T^{e}, P^{e}, Q^{e}\right)=\left(S^{e}, \frac{\tau S^{e}}{\mu}, I^{e}, \frac{\sigma I^{e}}{\mu+\delta+\rho_{f}+v}, \frac{\eta I^{e}+\nu F^{e}}{\mu+\rho_{t}}, \frac{Z}{\mathscr{R}_{r}^{m}}, P^{e}\left(\mathscr{R}_{r}^{m}-1\right)\right)
$$

where

$$
\begin{aligned}
S^{e} & =\frac{\mu N}{\beta I^{e}+\alpha Q^{e}+\mu+\tau}, \\
I^{e} & =\frac{-b+\sqrt{b^{2}-4 a c}}{2 a}, \\
a & =\beta(\mu+\rho+\eta+\sigma), \\
b & =(\mu+\rho+\eta+\sigma)\left(\tau+\mu+\alpha Q^{e}\right)-\mu \beta N, \\
c & =-\alpha \mu Q^{e} N .
\end{aligned}
$$

The results of the stability analysis about the endemic equilibrium of the multiple control model (45) are summarized in the theorem below.

Theorem 13. The endemic equilibrium (51) is locally asymptotically stable whenever $\mathscr{R}_{h}^{m}=\left(N / S^{e}\right)>1$ and $\mathscr{R}_{r}^{m}>1$.

Proof. For $\mathscr{R}_{h}^{m}=\left(N / S^{e}\right)>1$ and $e=1$, the Jacobian of the multiple control model (45) evaluated at EE (51) is

$$
J^{*}=\left(\begin{array}{ccccccc}
a_{11} & 0 & a_{13} & 0 & 0 & 0 & -\alpha S^{*} \\
\tau & -\mu & 0 & 0 & 0 & 0 & 0 \\
a_{31} & 0 & 0 & 0 & 0 & 0 & \alpha S^{*} \\
0 & 0 & \eta & \nu & -\left(\mu+\rho_{t}\right) & 0 & 0 \\
0 & 0 & 0 & 0 & 0 & a_{66} & a_{67} \\
0 & 0 & 0 & 0 & 0 & a_{76} & 0
\end{array}\right) .
$$

The eigenvalues of the Jacobian are

$$
\begin{aligned}
& \lambda_{1}=-\mu, \\
& \lambda_{2}=-\left(\delta+\mu+\nu+\rho_{f}\right), \\
& \lambda_{3}=-\left(\mu+\rho_{t}\right), \\
& \lambda_{4,5}=\frac{a_{11} \pm \sqrt{a_{11}^{2}+4 a_{13} a_{31}}}{2}, \\
& \lambda_{6,7}=\frac{a_{66} \pm \sqrt{a_{66}^{2}+4 a_{67} a_{76}}}{2},
\end{aligned}
$$


where $a_{11}=-\left(\mu+\tau+\beta I^{e}+\alpha Q^{e}\right), a_{13}=-\beta S^{e}, a_{31}=\beta I^{e}+$ $\alpha Q^{e}, a_{66}=-\left(\xi+(1-c) \phi Q^{e}\right), a_{67}=-(1-c) \phi P^{e}$, and $a_{76}=$ $(1-c) \phi Q^{e}$. Clearly, we can see that all the eigenvalues have negative real parts. This completes the proof.

This result implies that if $\mathscr{R}_{0}^{m}>1$, the disease will persist in the population when multiple controls are considered as a control measure. A possible explanation for this could be that the control measures are not properly implemented.

\section{Optimal Control Problem}

We have been able to show that the best control measure is the multiple control intervention technique for reducing the spread of Lassa fever, but it is an obvious fact that some communities or areas where this disease occurs may be faced with limited resources needed in combating the menace.
Hence, we decided to consider a better control intervention scheme that will not just reduce or eradicate the disease but also carry out the task with minimum cost [33]. Optimal control theory is a mathematical tool that has been successfully used to analyze such problem and is considered here. In an attempt to reduce the cost of implementing the multiple control intervention technique (external protection, isolation, treatment, and rodent control), we take the control parameters $\tau, \sigma, \eta$, and $c$ in the multiple control model (45) as measurable functions of time $t$ and then formulate an appropriate optimal control functional that minimizes the cost of implementing the controls subject to the model. For simplicity, we let $\tau=u_{1}(t), \sigma=u_{2}(t)$, $\eta=u_{3}(t)$, and $c=u_{4}(t)$. This implies that the multiple control scheme is said to be optimal if it minimizes the objective functional

$$
\mathscr{J}\left(u_{1}, u_{2}, u_{3}, u_{4}\right)=\int_{0}^{t_{f}}\left[A_{1} S(t)+A_{2} I(t)+B_{1} P(t)+B_{2} Q(t)+C_{1} u_{1}^{2}(t)+C_{2} u_{2}^{2}(t)+C_{3} u_{3}^{2}(t)+C_{4} u_{4}^{2}(t)\right] \mathrm{d} t
$$

subject to the multiple control model (45), where $t_{f}$ is the final time and the coefficients, $A_{1}, A_{2}, B_{1}, B_{2}, C_{1}, C_{2}, C_{3}, C_{4}$ are known as balancing coefficients. This procedure has to do with minimizing the number of susceptible humans, infected humans, susceptible rodents, and infected rodents, as well as cost of applying the controls. To take into account the nonlinearity that might arise from the controls, we make use of quadratic functions in measuring the cost control $[5,7-11,33]$. The existence of the optimal control quadruple $\left(u_{1}^{*}(t), u_{2}^{*}(t), u_{3}^{*}(t), u_{4}^{*}(t)=u^{*}(t)\right)$ that minimizes our objective functional (55) subject to the state system which is the multiple control model (45) comes from Fleming and Rishel [46]:

$$
J\left(u^{*}(t)\right)=\min \left\{\mathbb{J}: u(t) \in \mathbb{U}, t \in\left[0, t_{f}\right]\right\}
$$

where

$$
u(t)=\left(u_{1}(t), u_{2}(t), u_{3}(t), u_{4}(t)\right)
$$
$\mathbb{U}=\{u(t): u(t)$ are measurable, $0 \leq u(t) \leq 1\}$ is the control set. Pontryagin's maximum principle [47] brings up functions that are termed as adjoint functions that help us join the state system (45) with the objective functional (55). We aim to use this principle in minimizing a Hamiltonian $H$, by converting the minimization problem of the objective functional state system, with respect to $u_{1}(t), u_{2}(t), u_{3}(t)$, and $u_{4}(t)$. The Hamiltonian for the objective functional (55) and the state system is given by

$$
\begin{aligned}
H= & A_{1} S(t)+A_{2} I(t)+B_{1} P(t)+B_{2} Q(t)+C_{1} u_{1}^{2}(t)+C_{2} u_{2}^{2}(t)+C_{3} u_{3}^{2}(t)+C_{4} u_{4}^{2}(t) \\
& +\lambda_{s}(\mu N(t)-\beta S(t) I(t)-\alpha S(t) Q(t)-(\mu+\tau) S(t))+\lambda_{G}\left(\tau S(t)-(1-e) \phi_{h 1} G(t) I(t)-\mu G(t)\right) \\
& +\lambda_{I}\left(\beta S(t) I(t)+\alpha S(t) Q(t)+(1-e) \phi_{h 1} G(t) I(t)-(\mu+\rho+\eta+\sigma) I(t)\right)+\lambda_{F}\left(\sigma I(t)-\left(\mu+\delta+\rho_{f}+v\right) F(t)\right) \\
& +\lambda_{T}\left(\eta I(t)+v F(t)-\left(\mu+\rho_{t}\right) T(t)\right)+\lambda_{R}\left(\rho I(t)+\rho_{t} T(t)+\rho_{f} F(t)-\mu R(t)\right) \\
& +\lambda_{P}(\mu Z(t)-\phi(1-c) P(t) Q(t)-\mu P(t))+\lambda_{Q}(\phi(1-c) P(t) Q(t)-\mu Q(t)),
\end{aligned}
$$

where $\lambda_{S}, \lambda_{G}, \lambda_{I}, \lambda_{F}, \lambda_{T}, \lambda_{R}, \lambda_{P}$, and $\lambda_{Q}$ are associated adjoints for the states $S, G, I, F, T, R, P$, and $Q$, respectively. Given an optimal control quadruple $\left(u_{1}^{*}(t), u_{2}^{*}(t), u_{3}^{*}(t), u_{4}^{*}(t)\right)$ together with corresponding states $\left(S^{*}, G^{*}, I^{*}, F^{*}, T^{*}\right.$, $\left.R^{*}, P^{*}, Q^{*}\right)$ that minimizes $J\left(u_{1}, u_{2}, u_{3}, u_{4}\right)$ over $\mathbb{U}$, there exists adjoint variables $\lambda_{S}, \lambda_{G}, \lambda_{I}, \lambda_{F}, \lambda_{T}, \lambda_{R}, \lambda_{P}$, and $\lambda_{Q}$ satisfying 


$$
\begin{aligned}
& \dot{\lambda}_{S}=-A_{1}+\lambda_{S}\left(\beta I+\alpha Q+\left(\mu+u_{1}\right)-\lambda_{G} u_{1}-\lambda_{I}(\beta I+\alpha Q)\right. \\
& \dot{\lambda}_{G}=\lambda_{G}[(1-e) \beta I+\mu]+\lambda_{I}[(1-e) \beta I] \\
& \dot{\lambda}_{I}=-A_{2}+\lambda_{S} \beta S+\lambda_{G}(1-e) \beta G+\lambda_{I}\left(-\beta S-(1-e) \beta E+\left(\mu+\rho+u_{3}+u_{2}\right)\right)-\lambda_{F} u_{2}+\lambda_{T} u_{3}-\lambda_{R} \rho \\
& \dot{\lambda}_{F}=\lambda_{F}\left(\mu+\delta+\rho_{f}+v\right)-\lambda_{T} v-\lambda_{R} \rho_{f} \\
& \dot{\lambda}_{T}=\lambda_{T}\left(\mu+\rho_{t}\right)-\lambda_{R} \rho_{t} \\
& \dot{\lambda}_{R}=\lambda_{R} \mu \\
& \dot{\lambda}_{P}=-B_{1}+\lambda_{P}\left(\left(1-u_{4}\right) \phi Q+\mu\right)-\lambda_{Q}\left(\phi\left(1-u_{4}\right) Q\right) \\
& \dot{\lambda}_{Q}=-B_{2}+\lambda_{S} \alpha S-\lambda_{I} \alpha S+\lambda_{P}\left(\phi\left(1-u_{4}\right) P\right)+\lambda_{Q}\left(\mu-\phi\left(1-u_{4}\right) P\right)
\end{aligned}
$$

together with transversality conditions $\lambda_{k}\left(t_{f}\right)=$ 0 , for $k=S, G, I, F, T, R, P$, and $Q$.

Note that we get the differential equation (58) which governs the adjoint variables by differentiating the appropriate Hamiltonian function (57) with respect to the corresponding states as follows:

$$
-\frac{\mathrm{d} \lambda_{k}}{\mathrm{~d} t}=\frac{\mathrm{d} H}{\mathrm{~d} k} .
$$

Now, consider the optimality conditions

$$
\begin{aligned}
& 0=\frac{\partial H}{\partial u_{1}}, \\
& 0=\frac{\partial H}{\partial u_{2}}, \\
& 0=\frac{\partial H}{\partial u_{3}}, \\
& 0=\frac{\partial H}{\partial u_{4}} .
\end{aligned}
$$

So, for $u_{1}$, we have

$$
2 C_{1} u_{1}^{*}-\lambda_{S} S(t)+\lambda_{G} S(t)=0 .
$$
have

Then, solving for $u_{1}$ in the optimality conditions, we

$$
u_{1}^{*}=\frac{S(t)\left(\lambda_{S}-\lambda_{G}\right)}{2 C_{1}},
$$

and subsequently taking bounds into consideration, we have

$$
u_{1}^{*}=\min \left\{0, \max \left\{\frac{S(t)\left(\lambda_{S}-\lambda_{G}\right)}{2 C_{1}}, 1\right\}\right\} .
$$

Solving for $u_{2}$ in the optimality conditions, we have

$$
u_{2}^{*}=\frac{I(t)\left(\lambda_{I}-\lambda_{F}\right)}{2 C_{2}},
$$

and subsequently taking bounds into consideration, we have

$$
u_{2}^{*}=\min \left\{0, \max \left\{\frac{I(t)\left(\lambda_{I}-\lambda_{F}\right)}{2 C_{2}}, 1\right\}\right\} .
$$

Similarly, we obtain that

$$
\begin{aligned}
& u_{3}^{*}=\min \left\{0, \max \left\{\frac{I(t)\left(\lambda_{I}-\lambda_{T}\right)}{2 C_{3}}, 1\right\}\right\}, \\
& u_{4}^{*}=\min \left\{0, \max \left\{\frac{\phi P Q\left(\lambda_{P}-\lambda_{Q}\right)}{2 C_{4}}, 1\right\}\right\} .
\end{aligned}
$$

These results demonstrate that there exists an optimal control quadruple $\left(u_{1}^{*}, u_{2}^{*}, u_{3}^{*}, u_{4}^{*}\right)$ which decreases the spread of Lassa fever disease considering the multiple control intervention strategy with minimum cost. Since the optimal control quadruple is ascertained to be dependent on parameter, we carry out further analysis using numerical simulation to reveal more details on the trajectory of the optimal control using published data. The numerical simulation of the optimal control is presented in the next section.

\section{Numerical Simulations}

In this section, we present numerical simulations to support our analytical predictions. The parameter values for the numerical simulations are given in Table 1.

From Figure 1(a), we discover that when $\mathscr{R}_{0}$ (which stands for the basic reproduction number of the control-free model) is greater than 1, the susceptible humans and susceptible rodents decrease, while the infected humans and rodents increase. This shows that the disease persists in the human and rodent population and thus agrees with Theorem 3 that states that endemic equilibrium in the absence of the control measures is stable provided that $\mathscr{R}_{0}$ is greater than unity.

Next, from Figure 1(b), we discover that when $\mathscr{R}_{0}$ is less than unity, the infected humans and rodents decrease. Particularly, the infected rodents, which are the source of the virus, witnessed drastic decrease, and this agrees with Theorem 1 which says that the DFE of the model in the absence of control measures is stable provided $\mathscr{R}_{0}$ is less than unity.

From Figure 2, we investigate the impact of the control measures on the models by comparing the no control model with the multiple control model. It is seen that the susceptible humans decrease faster in the presence of multiple control strategies. This supports our target of reducing human susceptibility to Lassa fever disease. Also, the infected humans were reduced almost to zero, which shows 
TABle 1: Parameter values used for numerical simulations.

\begin{tabular}{lcc}
\hline Parameters & Parameter values & \\
\hline$\mu$ & 0.0000457 & - \\
$\beta$ & 0.00002 & - \\
$\alpha$ & 0.00001 & - \\
$\rho_{\xi}$ & 0.0476 & - \\
$\phi$ & 0.2 & - \\
$\tau$ & 0.002 & - \\
$e$ & 0.07 & - \\
$\eta$ & 0.85 & {$[49]$} \\
$\sigma$ & 0.005 & - \\
$\delta$ & 0.057 & - \\
$\rho_{f}$ & 0.333 & - \\
$v$ & 0.0376 & - \\
$\rho_{t}$ & 0.03 & - \\
$c$ & 0.1184 & - \\
\hline
\end{tabular}
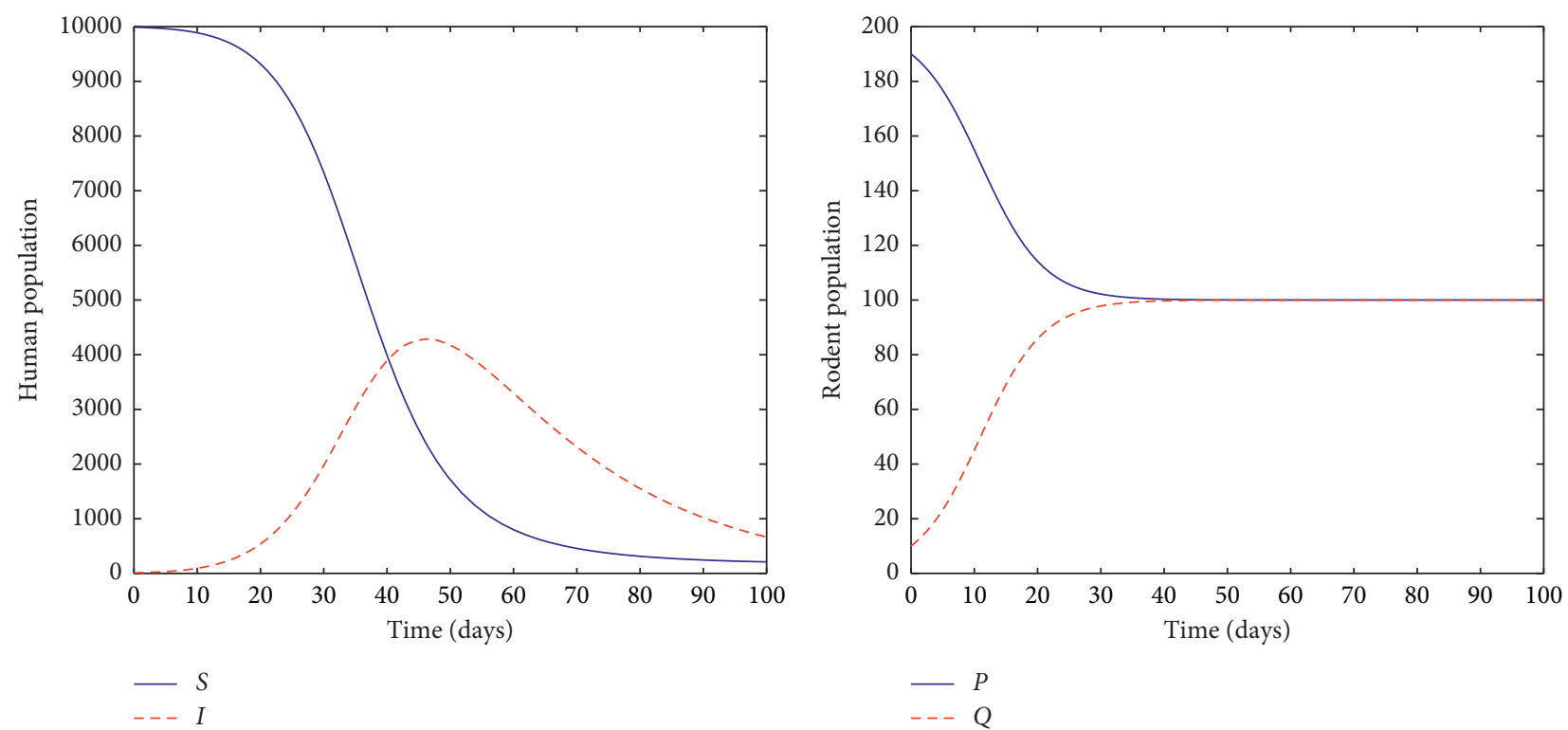

(a)
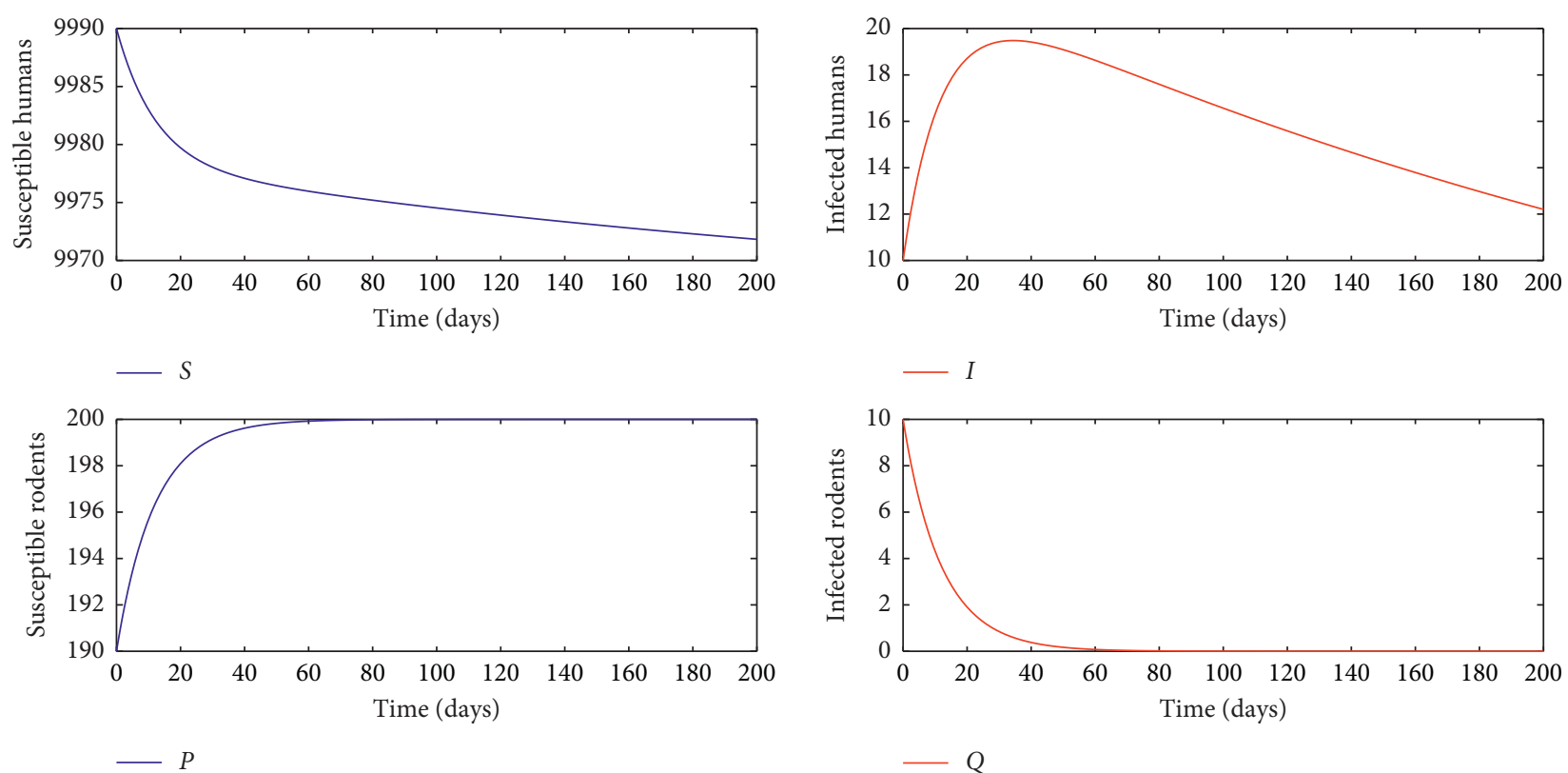

(b)

FIGURE 1: Graphical illustration of possible dynamics of model (1) for $\mathscr{R}_{0}$, less than unity and greater than unity: (a) $\mathscr{R}_{h}=4.1968$ and $\mathscr{R}_{r}=2.0000$; (b) $\mathscr{R}_{h}=0.3957$ and $\mathscr{R}_{r}=0.6000$. 


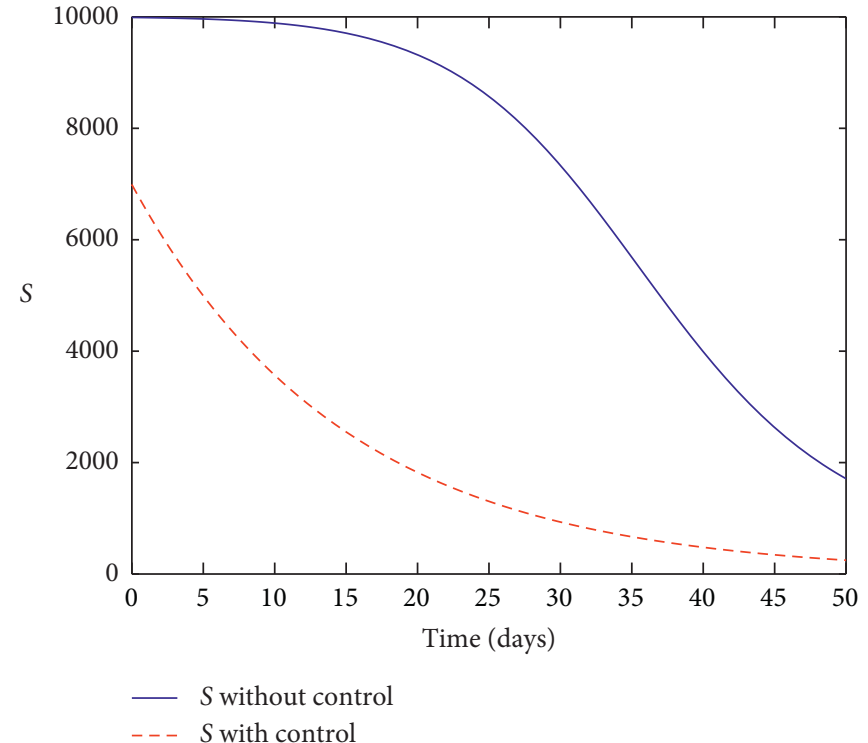

(a)

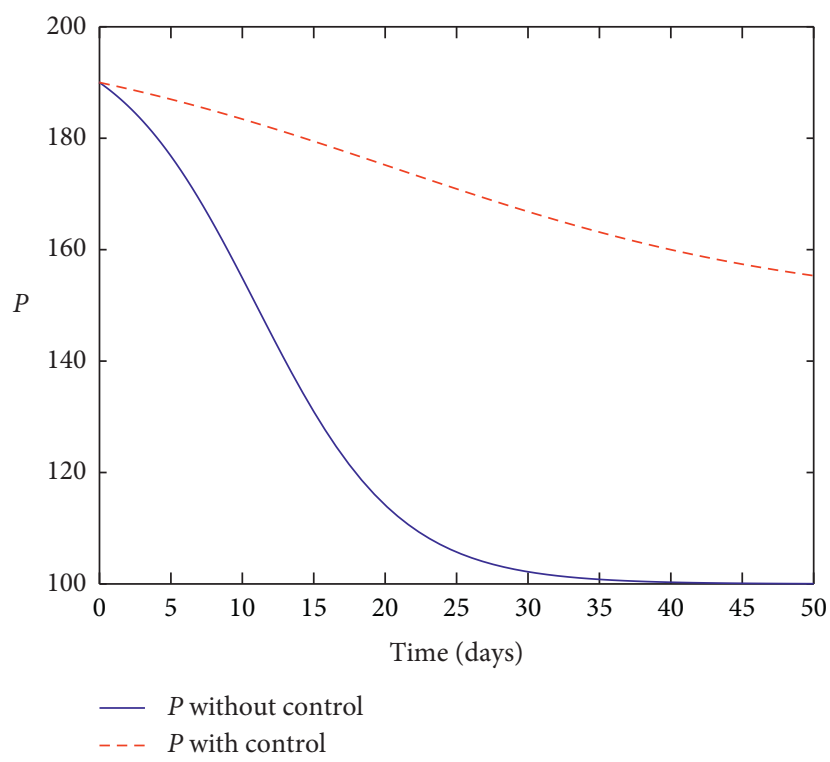

(c)

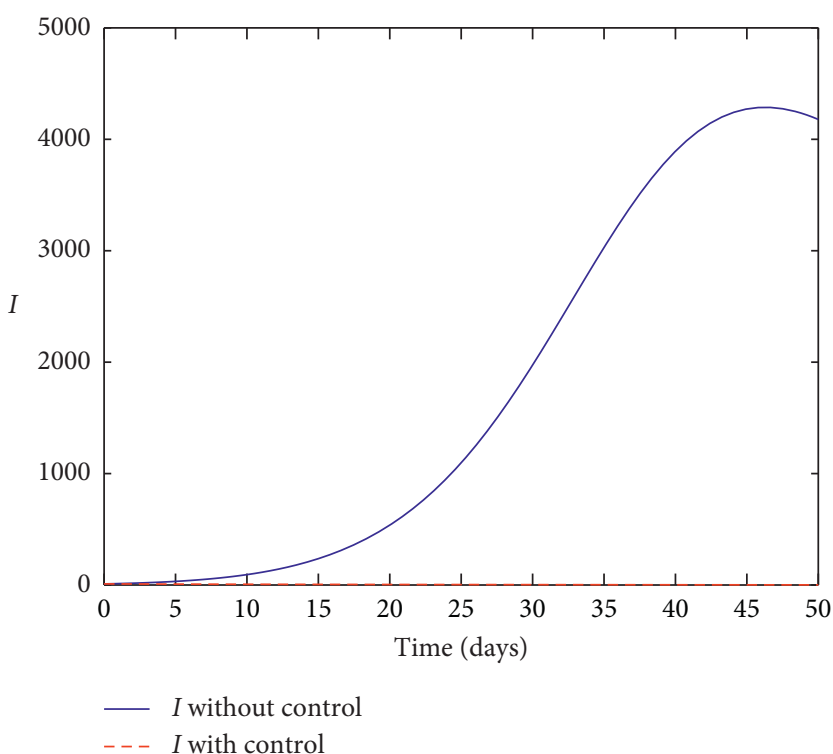

(b)

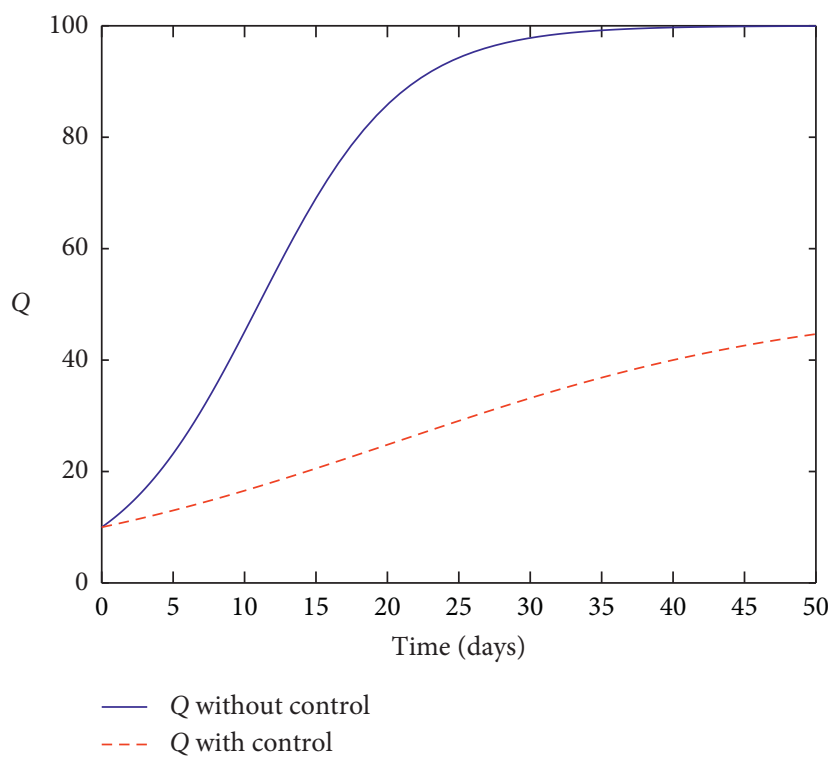

(d)

FIgURE 2: Graphical illustration of possible dynamics of model (1) and multiple control model (45).

that multiple control measures can eliminate infected humans with Lassa fever, which agrees with intuitive expectation. Next, in the rodent compartment, we observed that the susceptible rodents were reduced, but not as compared with susceptible humans. A possible explanation could be because there was only one control measure that was introduced in the rodent compartment. But we can also see that infected rodents reduced with the introduction of control. By this we can say that our result agrees with real life expectation; therefore, this model can be used to study real life situation. The results can be used to advice policy makers in taking decisions on how to reduce the incidence of Lassa fever in the society.
From Figure 3, we look at the long time dynamics. We compare model (1) which is the model without control and model (45) which is the multiple control model. We discovered that with the multiple control measures, infected humans get eliminated faster. This result agrees with Theorem 12. Also, with longer duration, we can see that the infected rodents reduce more with control than without control, which also agrees with our earlier theorem. This implies that for the rodents to die out completely, more effective control strategies will be required.

As earlier stated, the optimal control measures $\left(u_{1}^{*}(t), u_{2}^{*}(t), u_{3}^{*}(t), u_{4}^{*}(t)\right)$ are parameter dependent. So, in order to determine their magnitudes, we resort to numerical 

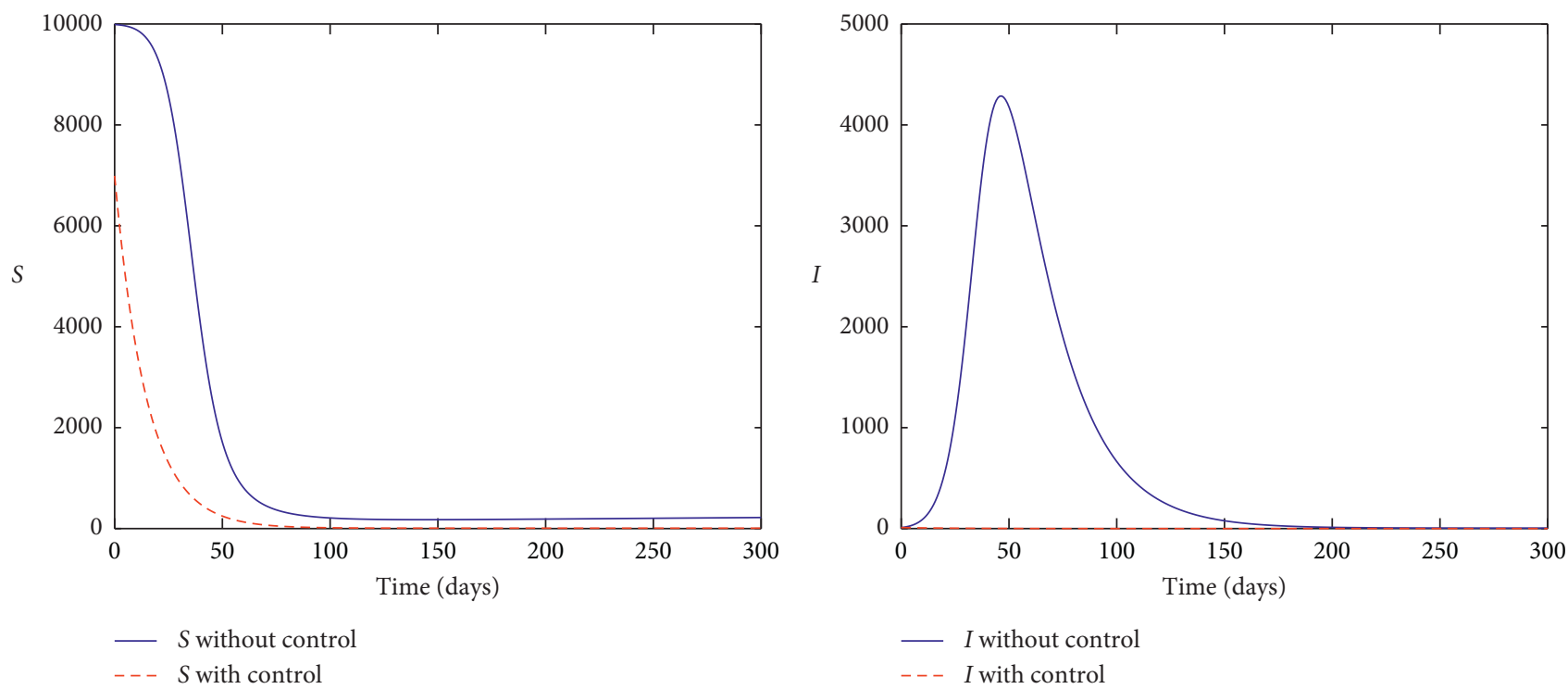

(a)

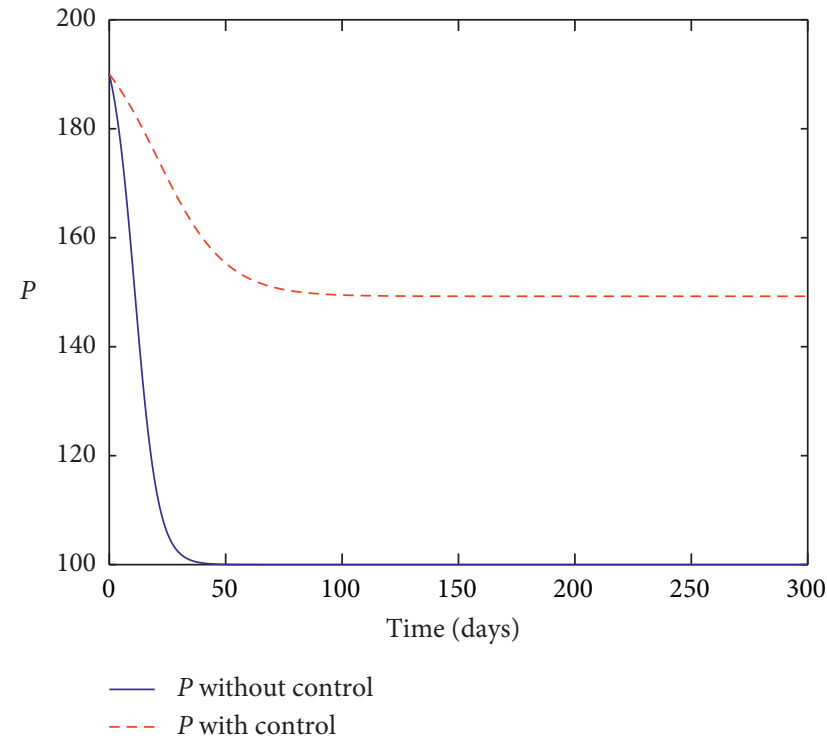

(c)

(b)

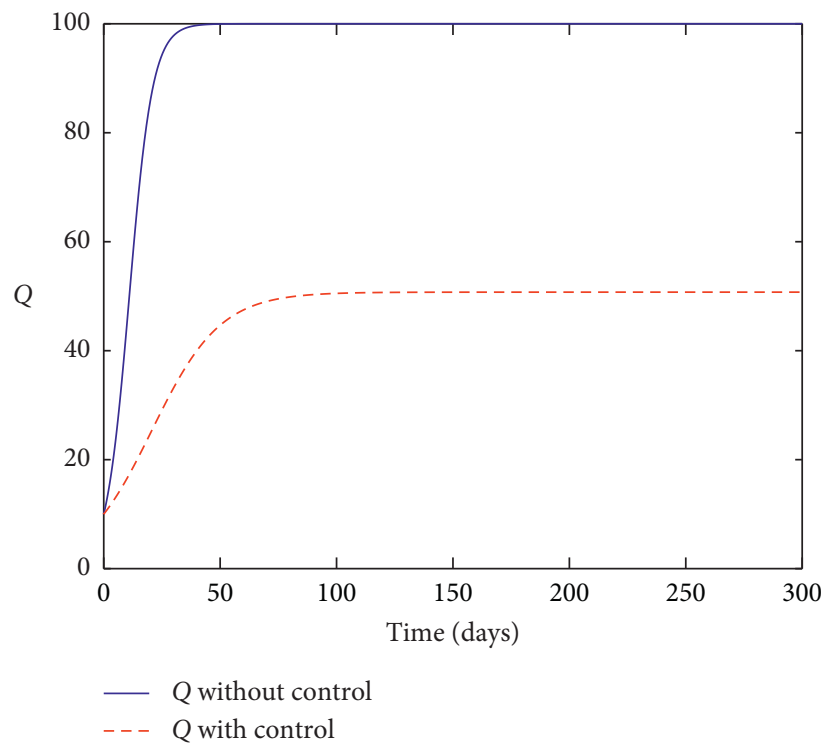

(d)

FIGURE 3: Graphical illustration of possible dynamics of model (1) and multiple control model (45).

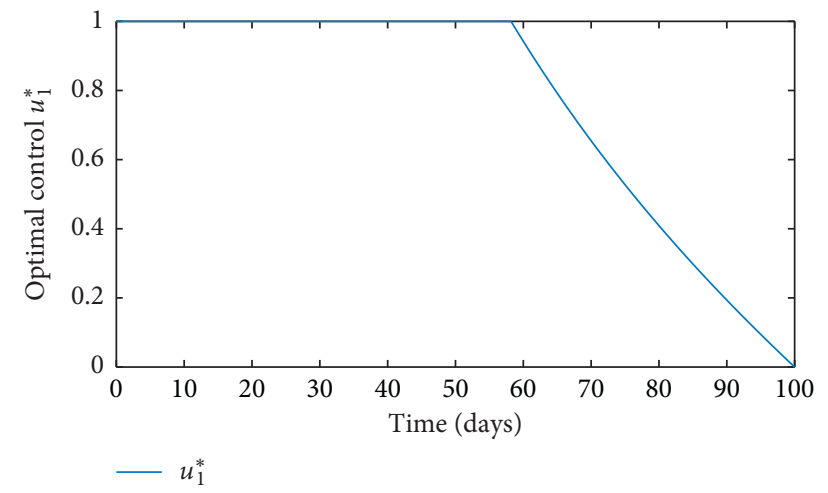

(a)

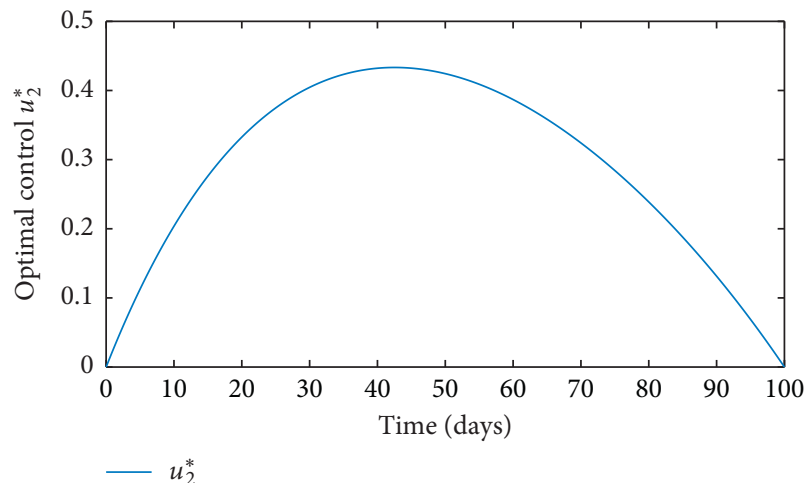

(b)

FIgURE 4: Continued. 


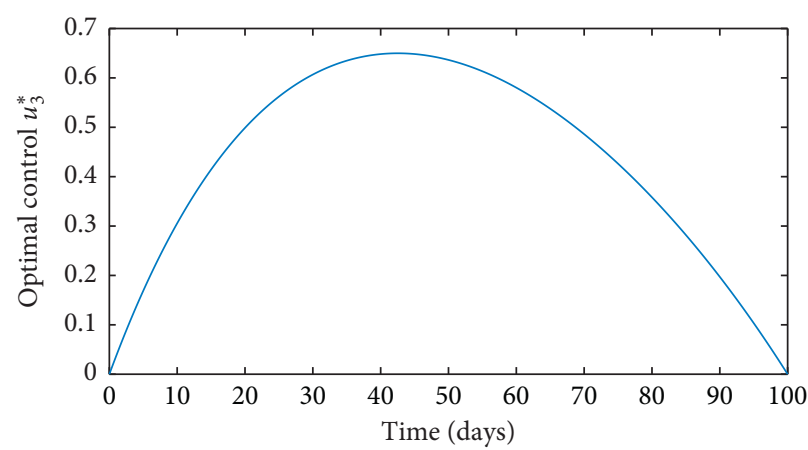

$-u_{3}^{*}$

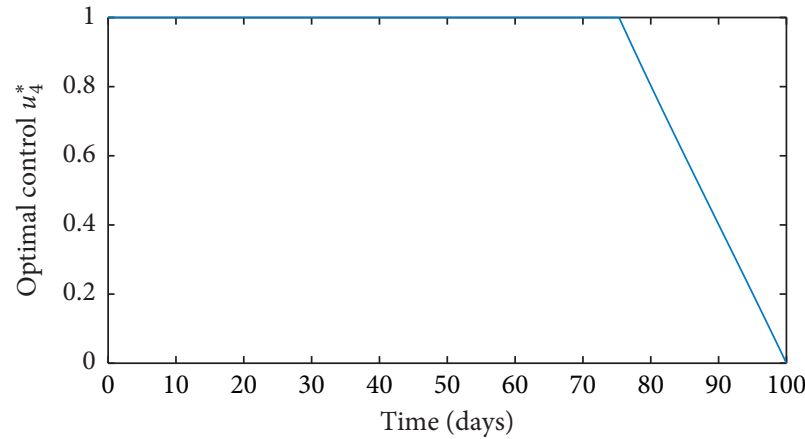

$-u_{4}^{*}$

(c)

(d)

FIgURE 4: Graphical illustration showing the optimal control functions $\left(u_{1}^{*}(t), u_{2}^{*}(t), u_{3}^{*}(t)\right.$, and $\left.u_{4}^{*}(t)\right)$.

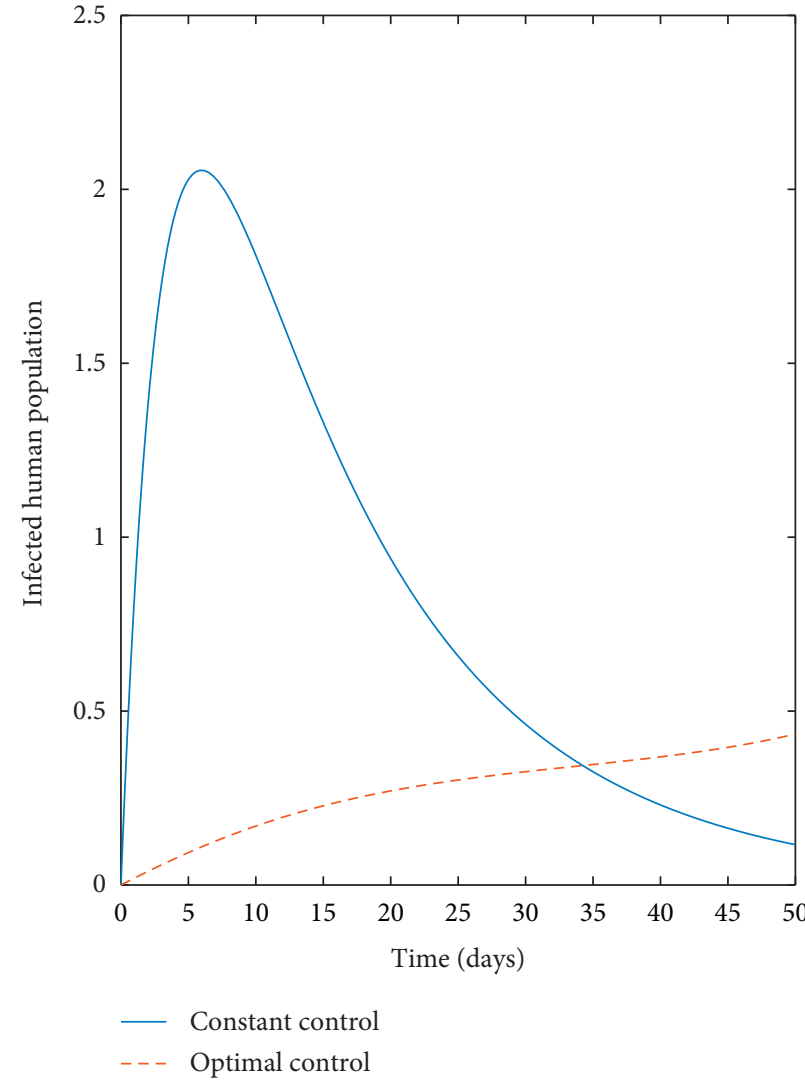

(a)

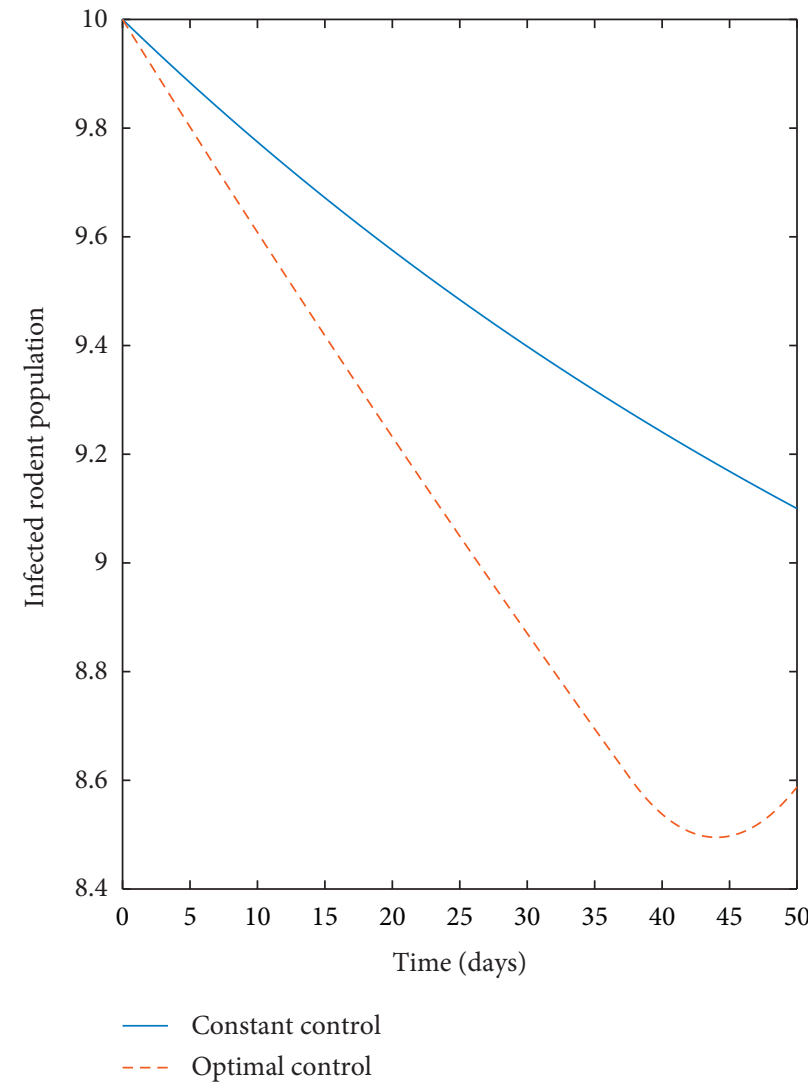

(b)

FIGURE 5: Graphs showing the relationship that exists between the state variables of our model: with constant control and optimal control at short term.

simulations. This was carried out with the parameter values given in Table 1 with their assigned cost factors for illustration. We consider the forward backward algorithm of [32] to obtain the optimal control functions $\left(u_{1}^{*}(t), u_{2}^{*}(t)\right.$, $\left.u_{3}^{*}(t), u_{4}^{*}(t)\right)$ that minimize the cost functional, and they are shown in Figure 4 . The figures suggest that it is optimal to begin introducing control techniques from the beginning of Lassa fever disease invasion and continue with maximal effort until the disease is eradicated. This is realistic, since controlling the disease at the onset will stop it from affecting the entire population.

We show a simple illustration of the impact of the optimal control functions $\left(u_{1}^{*}(t), u_{2}^{*}(t), u_{3}^{*}(t), u_{4}^{*}(t)\right)$ on the infected class of our multiple control model in Figures 5 and 6. These figures are obtained by comparing the 


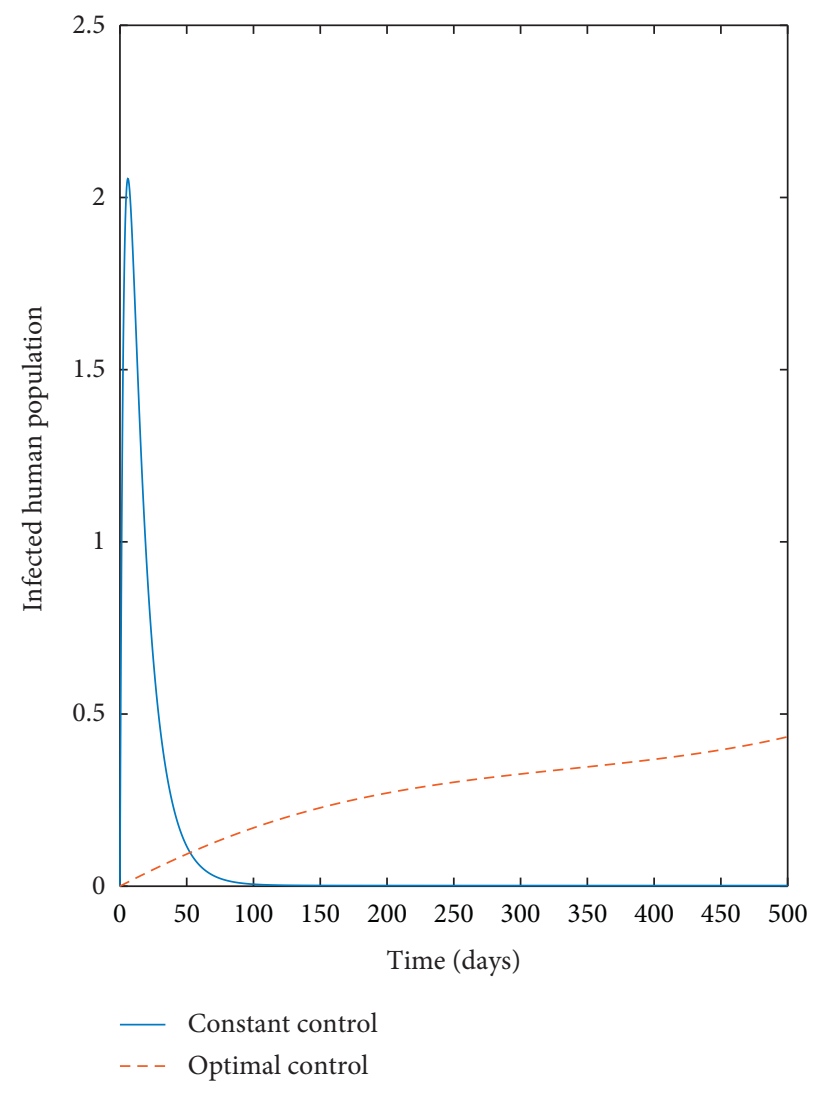

(a)

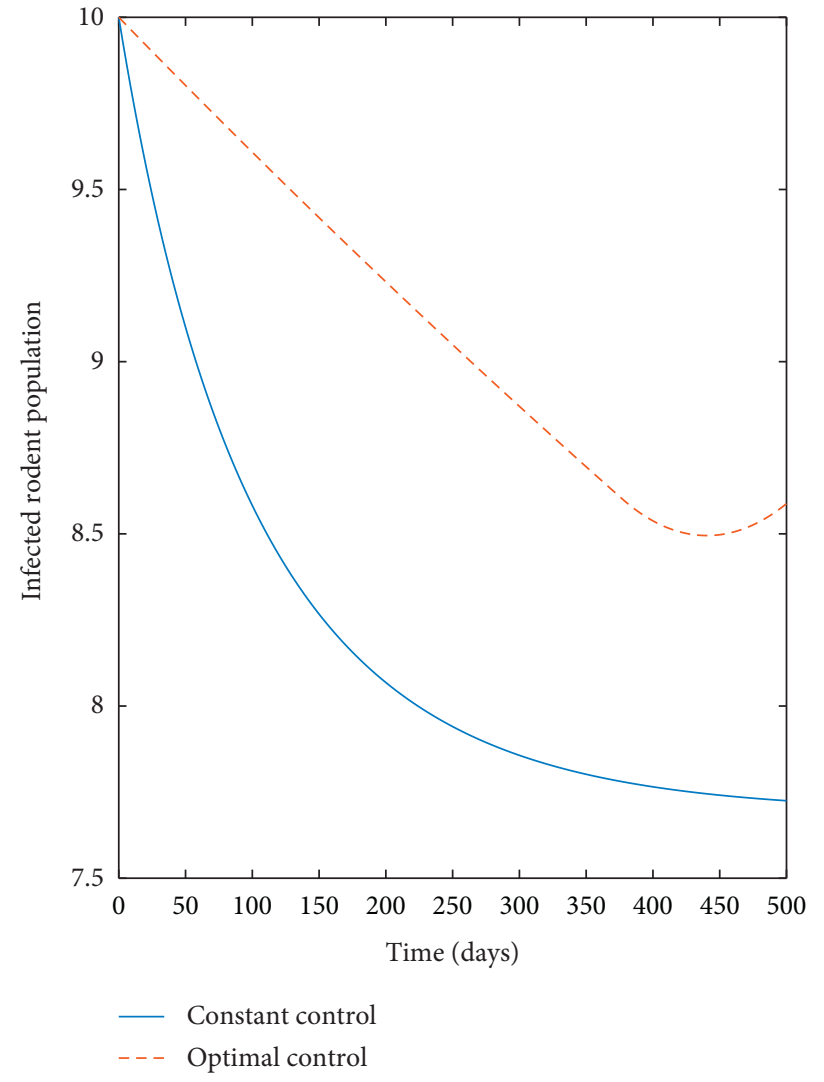

(b)

FIGURE 6: Graphs showing the relationship that exists between the state variables of our model: with constant control and optimal control at a longer duration.

numerical solution of our model when multiple control is introduced and when optimal control is introduced, both at short term and at longer term. From the figures, we observe that the optimal control tends to do better or have greater effect at the initial time, but as time increases, we observe that the constant control had a better and effective impact on the infectious class of our model. This agrees with intuitive expectation, as constant control yields better results. This is because it does not consider cost. We conclude that for poor communities, optimal control may be feasible, but for richer communities, constant control will be the best option.

\section{Discussion}

We explored the control intervention measures of the dynamics of Lassa fever in a population of humans and rats that are homogeneously mixed together. Vital information about the Lassa fever dynamics was obtained from our analysis, and choosing an appropriate mathematical epidemiological model will play an important role in giving all possible information on the general dynamics of the disease.

We have been able to show that it is possible for Lassa fever to be eradicated from the entire population, if we can reduce the basic reproduction number $\mathscr{R}_{0}$ to a value that is less than unity. This can happen if the infected humans begin exhibiting healthy living practices, which include keeping off from areas infected by rats or areas close to waste bin and by keeping their environment clean and tidy and reducing any practice that will attract infected rats in their places of abode. On the other hand, if $\mathscr{R}_{0}>1$, an outbreak which grows at a rate $\lambda^{+}$might occur. This outbreak will persist in the population, since the endemic equilibrium is globally asymptotically stable, unless control intervention measures are introduced into the system.

Next, we investigated the benefits of some control intervention measures like external protection, isolation, treatment, rodent control, and the multiple control intervention strategies by the extension of the control-free model earlier formulated. We computed the basic reproduction numbers for external protection, isolation, treatment, rodent control, and multiple control intervention measure models which are given, respectively, as $\mathscr{R}_{0}^{e}, \mathscr{R}_{0}^{f}$, $\mathscr{R}_{0}^{t}, \mathscr{R}_{0}^{c}$, and $\mathscr{R}_{0}^{m}$. Analyses of our models have shown that external protection, isolation, treatment, rodent control, and multiple control intervention technique reduce the number of secondary infections. Further analyses also revealed that using the multiple intervention technique demonstrates the highest effect in reducing the number of secondary infections, followed by isolation, external protection, and then treatment. We further showed that Lassa fever can be quickly eradicated by any of these control 
intervention strategies provided that the corresponding basic reproduction number is less than one. However, if the control is not effective enough such that $\mathscr{R}_{0}^{e}>1, \mathscr{R}_{0}^{f}>1$, $\mathscr{R}_{0}^{t}>1, \mathscr{R}_{0}^{c}>1$, or $\mathscr{R}_{0}^{m}>1$, then the disease will persist in the population.

Furthermore, we focused on analyzing the multiple control model since it is evident from our analyses that it is the best method when compared with the single control and even the control-free models. We investigated the best strategy to minimize the spread of Lassa fever using the multiple control with a minimum cost. Analyzing an appropriate optimal control cost functional subject to the multiple control model, we obtained an optimal control quadruple $\left(u_{1}^{*}, u_{2}^{*}, u_{3}^{*}, u_{4}^{*}\right)$ that reduces the spread of infections with a minimum cost. The results of our optimal control analysis revealed that it is optimal to treat individuals immediately as they get infected and begin to use external protection and isolate infected as soon as the outbreak starts and continue with maximal effort until the outbreak ends.

The dynamical behavior of our models agree with the intuitive expectation of Lassa fever dynamics in real life. Thus, the models can be used to predict future evolution of Lassa fever in communities where the disease is endemic. It can also be used to study how to control Lassa fever with minimum cost using control intervention techniques like external protection, isolation, treatment, and rodent control.

Having provided new insights into the dynamics and control intervention strategies for Lassa fever disease in a homogeneous mixed population setting, it is important to note that this study is not without some limitations. Firstly, in our assumption, we studied an assumed constant population. But we know that this case is not necessarily true because in real life there are always births and deaths which are not necessarily equal especially also with outbreaks that last for a long period of time. We also assumed homogeneity in disease transmission, but it is not always true since heterogeneity is an essential part of epidemiology and has been shown to have influence on disease transmission $[31,48]$. All these will be considered in further studies.

\section{Data Availability}

The data supporting this deterministic model are from previously published articles and they have been duly cited in this paper.

\section{Conflicts of Interest}

The authors declare that there are no conflicts of interest regarding the publication of this paper.

\section{References}

[1] World Health Organisation (WHO), November, 2018.

[2] Centres for Disease Control and Prevention (CDC), (November, 2018).
[3] World Health Organization (WHO), 2020, https://www.who. int/csr/don/20-february-2020-lassa-fever-nigeria/en/.

[4] O. C. Collins and K. J. Duffy, "analysis and optimal control intervention strategies of a waterborne disease model: a realistic case study," Journal of Applied Mathematics, vol. 2018, Article ID 2528513, 14 pages, 2018.

[5] O. C. Collins and K. S. Govinder, "Stability analysis and optimal vaccination of a waterborne disease model with multiple water sources," Natural Resource Modeling, vol. 29, no. 3, pp. 426-447, 2016.

[6] G. Zaman, Y. Han Kang, and I. H. Jung, "Stability analysis and optimal vaccination of an SIR epidemic model," BioSystems, vol. 93, no. 3, pp. 240-249, 2008.

[7] T. K. Kar and A. Batabyal, "Stability analysis and optimal control of an SIR epidemic model with vaccination," Biosystems, vol. 104, no. 2-3, pp. 127-135, 2011.

[8] K. W. Blayneh, A. B. Gumel, S. Lenhart, and T. Clayton, "Backward bifurcation and optimal control in transmission dynamics of West nile virus," Bulletin of Mathematical Biology, vol. 72, no. 4, pp. 1006-1028, 2010.

[9] F. B. Agusto, "Optimal chemoprophylaxis and treatment control strategies of a tuberculosis transmission model," World Journal of Model Simulations, vol. 5, no. 3, pp. 163-173, 2009.

[10] H. R. Joshi, "Optimal control of an HIV immunology model," Optimal Control Applications and Methods, vol. 23, no. 4, pp. 199-213, 2002.

[11] O. C. Collins and K. J. Duffy, "Optimal control of maize foliar diseases using the plants population dynamics," Acta Agriculturae Scandinavica, Section B-Soil \& Plant Science, vol. 66, no. 1, pp. 20-26, 2016.

[12] J. Mariën, B. Borremans, F. Kourouma et al., "Evaluation of rodent control to fight Lassa fever based on field data and mathematical modelling," Emerging Microbes \& Infections, vol. 8, no. 1, pp. 640-649, 2019.

[13] S. S. Musa, S. Zhao, D. Gao, Q. Lin, G. Chowell, and D. He, "Mechanistic modelling of the large-scale Lassa fever epidemics in Nigeria from 2016 to 2019," Journal of Theoretical Biology, vol. 493, p. 110209, 2020.

[14] S. Zhao, S. S. Musa, H. Fu, D. He, and J. Qin, "Large-scale Lassa fever outbreaks in Nigeria: quantifying the association between disease reproduction number and local rainfall," Epidemiology and Infection, vol. 148, pp. 1-12, 2020.

[15] G. L. Iacono, A. A. Cunningham, E. Fichet-Calvet et al., "Using modelling to disentangle the relative contributions of zoonotic and anthroponotic transmission: the case of Lassa fever," PLoS Neglected Tropical Diseases, vol. 9, no. 1, Article ID e3398, 2015.

[16] A. R. Akhmetzhanov, Y. Asai, and H. Nishiura, "Quantifying the seasonal drivers of transmission for Lassa fever in Nigeria," Philosophical Transactions of the Royal Society B: Biological Sciences, vol. 374, no. 1775, Article ID 20180268, 2019.

[17] E. A. Ilori, Y. Furuse, O. B. Ipadeola et al., "Epidemiologic and clinical features of Lassa fever outbreak in Nigeria, January 1may 6, 2018," Emerging Infectious Diseases, vol. 25, no. 6, pp. 1066-1074, 2019.

[18] E. A. Ilori, C. Frank, C. C. Dan-Nwafor et al., "Increase in Lassa fever cases in Nigeria, January-March 2018," Emerging Infectious Diseases, vol. 25, no. 5, pp. 1026-1027, 2019.

[19] N. A. Ajayi, C. G. Nwigwe, B. N. Azuogu et al., "Containing a Lassa fever epidemic in a resource-limited setting: outbreak description and lessons learned from Abakaliki, Nigeria 
(January-March 2012)," International Journal of Infectious Diseases, vol. 17, no. 11, pp. e1011-e1016.

[20] L. Roberts, "Nigeria hit by unprecedented Lassa fever outbreak," Science, vol. 359, no. 6381, pp. 1201-1202, 2018.

[21] B. M. Warner, D. Safronetz, and D. R. Stein, "Current research for a vaccine against Lassa hemorrhagic fever virus," Drug Design, Development and Therapy, vol. 12, pp. 2519-2527, 2018.

[22] I. S. Lukashevich, "The search for animal models for Lassa fever vaccine development," Expert Review of Vaccines, vol. 12, no. 1, pp. 71-86, 2013.

[23] I. S. Lukashevich and P. Pushko, "Vaccine platforms to control Lassa fever," Expert Review of Vaccines, vol. 15, no. 9, pp. 1135-1150, 2016.

[24] L. M. Branco, J. N. Grove, F. J. Geske et al., "Lassa virus-like particles displaying all major immunological determinants as a vaccine candidate for Lassa hemorrhagic fever," Virology Journal, vol. 7, no. 1, p. 279, 2010.

[25] R. A. Kenlyside, J. B. Mccormic, P. A. Webb, E. Smith, L. Elliot, and K. L. Johnson, "Case control study of mastomys natalensis and humans in Lassa virus-infected households in Sierra Lone," The American Journal of Tropical Medicine and Hygiene, vol. 32, pp. 829-837, 1983.

[26] A. J. Clayton, "Lassa immune serum," Bulletin of the World Health Organization, vol. 55, no. 55, pp. 435-439, 1977.

[27] O. Diekmann, H. Heesterbeek, and T. Britton, Mathematical Tools for Understanding Infectious Disease Dynamics, Vol. 7, Princeton University Press, Princeton, NJ, USA, 2012.

[28] I. M. Longini Jr., E. Ackerman, and L. R. Elveback, "An optimization model for influenza A epidemics," Mathematical Biosciences, vol. 38, no. 1-2, pp. 141-157, 1978.

[29] M. Kretzschmar, S. van den Hof, J. Wallinga, and J. van Wijngaarden, "Ring vaccination and smallpox control," Emerging Infectious Diseases, vol. 10, no. 5, pp. 832-841, 2004.

[30] H. S. Rodrigues, M. T. T. Monteiro, and D. F. M. Torres, "Vaccination models and optimal control strategies to dengue," Mathematical Biosciences, vol. 247, pp. 1-12, 2014.

[31] O. C. Collins and K. S. Govinder, "Stability analysis and optimal vaccination of a waterborne disease model with multiple water sources," Natural Resource Modeling, vol. 29, no. 3, pp. 426-447, 2016.

[32] S. Lenhart and J. T. Workman, Optimal Control Applied to Biological Models, Chapman and Hall/CRC, Boca Raton, FL, USA, 2007.

[33] R. L. Miller Neilan, E. Schaefer, H. Gaff, K. Renee Fisher, and S. Lenhart, "Modeling optimal intervention strategies for cholera," Bulletin of Mathematical Biology, vol. 72, no. 8, pp. 2004-2018, 2010.

[34] I. B. Sule, I. B. Wada, A. A. Abubakar et al., Outbreak of a Highly Virulent Lassa Fever Virus in Kano State, Nigeria: An Investigation Report 2015-2016, International Journal of Scientific \& Engineering Research, vol. 8, no. 1, pp. 613-619, 2017.

[35] M. B. Abdullahi, U. C. Doko, and M. Mamuda, "Sensitivity analysis in a Lassa fever deterministic mathematical model," AIP Conference Proceedings, vol. 1660, no. 1, 2015.

[36] X. Liao, L. Q. Wang, and P. Yu, "Stability of Dynamical Systems," Monograph Series on Nonlinear Science and Complexity, Vol. 5, Elsevier, Amsterdam, Netherlands, 2007.

[37] R. Reiszig, J. LaSalle, and S. Lefschetz, "Stability by Liapunov's direct method with applications," ZAMM-Journal of Applied Mathematics and Mechanics/Zeitschrift für Angewandte Mathematik und Mechanik, vol. 42, no. 10-11, p. 514, 1962.
[38] P. Van den Driessche and J. Watmough, "Reproduction numbers and sub-threshold endemic equilibria for compartmental models of disease transmission," Mathematical Biosciences, vol. 180, no. 1-2, pp. 29-48, 2002.

[39] C. Castillo-Chavez, S. Blower, P. van den Driessche, D. Kirschner, and A. A. Yakubu, Mathematical Approaches for Emerging and Reemerging Infectious Diseases: An Introduction, Vol. 1, Springer Science and Business Media, Berlin, Germany, 2002.

[40] J. H. Tien and D. J. D. Earn, "Multiple Transmission pathways and disease dynamics in a waterborne pathogen model," Bulletin of Mathematical Biology, vol. 72, no. 6, pp. 1506-1533, 2010.

[41] J. Ma and D. J. D. Earn, "Generality of the final size formula for an epidemic of a newly invading infectious disease," Bulletin of Mathematical Biology, vol. 68, no. 3, pp. 679-702, 2006.

[42] J. M. Tchuenche, S. A. Khamis, F. B. Agusto, and S. C. Mpeshe, "Optimal control and sensitivity analysis of an influenza model with treatment and vaccination," Acta Biotheoretica, vol. 59, no. 1, pp. 1-28, 2011.

[43] P. B. Jahrling, R. A. Hesse, G. A. Eddy, K. M. Johnson, R. T. Callis, and E. L. Stephen, "Lassa virus infection of Rhesus Monkeys: pathogenesis and treatment with Ribavirin," Journal of Infectious Diseases, vol. 141, no. 5, pp. 580-589, 1980.

[44] T. P. Monath, Lassa Fever and Marburg Virus Disease, vol. 28, pp. 212-219, World Health Organization, Geneva, Switerzland, 1974.

[45] CDC, "Imported Lassa fever, New Jersey, Centres for disease control and prevention," Morbidity and Mortality Weekly Report, vol. 53, no. 38, pp. 894-897, 2004.

[46] W. H. Fleming and R. W. Rishel, "Deterministic and stochastic optimal control," Bulletin of the American Mathematical Society, vol. 82, no. 6, pp. 869-870, 1976.

[47] L. S. Pontryagin, V. G. Boltyanskii, R. V. Gamkrelidze, and E. F. Mishchenko, The Mathematical Theory of Optimal Processes in Russian, Joint Publications Research Service, Arlington, Tx, USA, 1961.

[48] S. L. Robertson, M. C. Eisenberg, and J. H. Tien, "Heterogeneity in multiple transmission pathways: modelling the spread of cholera and other waterborne disease in networks with a common water source," Journal of Biological Dynamics, vol. 7, no. 1, pp. 254-275, 2013.

[49] S. M. Garba and A. B. Gumel, "Mathematical recipe for HIV elimination in Nigeria," Journal of the Nigerian Mathematical Society, vol. 29, pp. 1-66, 2010. 